\title{
Ready-to-use Fourier domain templates for compact binaries inspiraling along moderately eccentric orbits
}

\author{
Srishti Tiwari ${ }^{1}$, Achamveedu Gopakumar ${ }^{1}$, Maria Haney ${ }^{2}$, and Phurailatapam Hemantakumar ${ }^{3}$ \\ ${ }^{1}$ Department of Astronomy and Astrophysics, Tata Institute of Fundamental Research, Mumbai 400005, India \\ ${ }^{2}$ Physik-Institut, Universität Zürich, Winterthurerstrasse 190, 8057 Zürich, Switzerland \\ ${ }^{3}$ Indian Institute of Technology, Mumbai 4000\%6, India
}

(Dated: June 5, 2019)

\begin{abstract}
We derive analytic expressions that provide Fourier domain gravitational wave (GW) response function for compact binaries inspiraling along moderately eccentric orbits. These expressions include amplitude corrections to the two GW polarization states that are accurate to the first postNewtonian (PN) order. Additionally, our fully 3PN accurate GW phase evolution incorporates eccentricity effects up to sixth order at each PN order. Further, we develop a prescription to incorporate analytically the effects of $3 \mathrm{PN}$ accurate periastron advance in the GW phase evolution. This is how we provide a ready-to-use and efficient inspiral template family for compact binaries in moderately eccentric orbits. Preliminary GW data analysis explorations suggest that our template family should be required to construct analytic inspiral-merger-ringdown templates to model moderately eccentric compact binary coalescence.

PACS numbers: 04.30.-w, 04.30.Tv
\end{abstract}

\section{INTRODUCTION}

Observations of GW events by the advanced LIGO and VIRGO GW interferometers are ushering in the era of GW astronomy 1, 2. These GW events include merging black hole $(\mathrm{BH})$ binaries and an inspiraling neutron star (NS) binary 3 - 9]. Several scenarios that include longlived (galactic) field binaries, star clusters, galactic nuclei and active galactic nuclei can produce these observed GW events [10 14]. Fortunately, it may be possible to extract valuable information about the astrophysical origins of GW events in the near future. This requires accurate GW measurements of the spin-orbit misalignment or the orbital eccentricities of these GW events [15 17]. Using both frequency and time domain inspiral-mergerringdown (IMR) waveforms, residual orbital eccentricities of the first two GW events were restricted to be below 0.15 when these binaries entered aLIGO frequency window 18, 19]. Strictly speaking, the so far detected GW events do not exhibit any observational signatures of residual orbital eccentricities and are faithfully captured by IMR templates associated with compact binaries merging along quasi-circular orbits.

However, there exists a number of astrophysical scenarios that can produce GW events with non-negligible eccentricities in the frequency windows of ground-based GW detectors. Dense star clusters like the ubiquitous globular clusters are the most promising sites to form aLIGO relevant compact binaries with non-negligible orbital eccentricities [20]. A recent realistic modeling of globular clusters that involve general relativistic few body interactions provided non-negligible fraction of $\mathrm{BH}$ binaries with eccentricities $>0.1$ as they enter the aLIGO frequency window 14, 21 25. Additionally, there exists a number of other astrophysical scenarios that can force stellar mass compact binaries to merge with orbital eccentricities. This include GW induced merger during hy- perbolic encounters between BHs in dense clusters 26 and mergers influenced by Kozai effect in few body systems as explored in many detailed investigations (see Ref. 27] and references therein). Further, a very recent investigation pointed out that less frequent binary-binary encounters in dense star clusters can easily produce eccentric compact binary coalescence [28]. These detailed investigations suggest that it may be reasonable to expect GW events with non-negligible orbital eccentricities in the coming years. Non-negligible orbital eccentricities may be helpful to improve the accuracy with a network of GW interferometers to constrain parameters of compact binary mergers 29, 30. Moreover, massive $\mathrm{BH}$ binaries in eccentric orbits are of definite interest to maturing Pulsar Timing Arrays and the planned Laser Interferometer Space Antenna (LISA) [31, 32.

There are different on-going investigations to model eccentric compact binary coalescence. These efforts aim to provide template families that model GWs from IMR phases of eccentric coalescence. The initial effort, detailed in Ref. 19, provided a time-domain IMR family that requires orbital eccentricity to be negligible during the merger phase. The inspiral part of the above waveform family was based on certain $x$-model, introduced in Ref. 33, that adapted GW phasing formalism of Refs. 34, 35. Additionally, a preliminary comparison with two numerical relativity (NR) waveforms was also pursued in Ref. [19. An improved version of the above family was presented in Ref. [36] that employed certain quasi-circular merger waveform and which can reproduce their NR simulations for any mass ratio below 4 . These waveform families are expected to model GWs from eccentric coalescence when initial eccentricities were usually below 0.2 . Very recently, another time domain IMR family was introduced in Ref. 37]. This detailed effort combined various elements from post-Newtonian, selfforce and black hole perturbation approaches in tandem 
with NR simulations to model GWs from moderately eccentric non-spinning $\mathrm{BH}$ binary coalescence. The resulting IMR waveforms were validated with many NR simulations for eccentric binary $\mathrm{BH}$ mergers lasting around ten orbits with mass ratios below 5.5 and initial eccentricities below 0.2. The eccentric binary $\mathrm{BH}$ coalescence is also explored in the framework of the Effective-OneBody (EOB) approach [38. A formalism to incorporate orbital eccentricity in the existing EOB approach to model quasi-circular compact binary coalescence is presented in Ref. 39]. Additionally, Ref. [40] presented an EOB waveform family that incorporated elements of $2 \mathrm{PN}$ accurate eccentric orbital description while comparing with few NR simulations for eccentric binary $\mathrm{BH}$ coalescence. In contrast, the LIGO Scientific Collaboration (LSC) adapted Ref. [41] that provided a crude IMR prescription to model GW signals from merging highly eccentric compact binaries. This was employed to probe the ability of few LSC algorithms to extract burst-like signals in the LIGO data 42. Further, some of us developed a ready-to-use 'effective eccentric variant' of IMRPhenomD waveform to constrain the initial orbital eccentricity of the GW150914 black hole binary. This was pursued to justify the assumption of binary evolution along circular orbits for the event [18. A crucial ingredient of the above IMR waveform family involved an eccentric version of TaylorF2 approximant that incorporated in its Fourier phase the leading-order eccentricity corrections up to $3 \mathrm{PN}$ order. The present paper provides fully analytic frequency domain interferometric response function $\tilde{h}(f)$ relevant for GW data analysis of nonspinning compact binaries inspiraling along moderately eccentric PN-accurate orbits.

Our computation is aimed at extending the widely used TaylorF2 approximant that provides analytic frequency domain GW templates for compact binaries inspiraling along quasi-circular orbits [43]. This waveform family employs the method of stationary phase approximation (SPA) to compute analytically Fourier transform of temporally evolving GW polarization states, $h_{\times}$and $h_{+}$, for quasi-circular inspirals. The popular LSC approximant provides fully analytic Fourier domain GW response function $\tilde{h}(f)$ that incorporates $3.5 \mathrm{PN}$-accurate Fourier phase [43. In other words, this approximant provides general relativistic corrections to GW phase evolution that are accurate to $(v / c)^{7}$ order beyond the dominant quadrupolar order, where $v$ is the orbital velocity. The present manuscript details our derivation of a fully analytic $\tilde{h}(f)$ with $3 \mathrm{PN}$-accurate Fourier phase with sixth order eccentricity contributions in terms of certain initial eccentricity at each PN order. Additionally, we include $1 \mathrm{PN}$-accurate amplitude corrections and the effect of $3 \mathrm{PN}$-accurate periastron advance on the Fourier phases.

To derive our eccentric approximant, we extend the post-circular scheme of Ref. [44] to higher PN orders. This scheme involves expanding the Newtonian accurate $h_{\times}$and $h_{+}$as a power series in orbital eccentricity that requires analytic solution to the classic Kepler equation. We extend such a Newtonian approach by invoking a recent effort to solve analytically PN-accurate Kepler equation in the small eccentricity limit 45]. This detailed computation also provided analytic $1 \mathrm{PN}$-accurate amplitude corrected expressions for $h_{\times}$and $h_{+}$as a sum over harmonics in certain mean anomaly $l$ of PN-accurate Keplerian type parametric solution [45]. Additionally, the above PN-accurate decomposition explicitly incorporated the effect of periastron advance on individual harmonics, numerically explored using PN description in Ref. [46. We combine such $1 \mathrm{PN}$-accurate amplitude corrected $h_{\times}$and $h_{+}$expressions that incorporated eccentricity contributions to sixth order at each PN order with the two beam pattern functions, $F_{\times}$and $F_{+}$, to obtain fully analytic time domain GW response function $h(t)$. Our eccentric TaylorF2 approximant is obtained by applying the method of stationary phase approximation to such an analytic $h(t)=F_{+} h_{+}+F_{\times} h_{\times}$expression.

To obtain analytic expressions for several Fourier phases at their associated stationary points of $h(t)$, we require additional $\mathrm{PN}$-accurate expressions. This involves deriving $3 \mathrm{PN}$-accurate expression for the time eccentricity $e_{t}$, present in the $3 \mathrm{PN}$-accurate Kepler Equation [47, as a bivariate expansion in terms of orbital angular frequency $\omega$, its initial value $\omega_{0}$ and $e_{0}$, the value of $e_{t}$ at $\omega_{0}$. This lengthy computation extends to $3 \mathrm{PN}$ order, the idea of certain asymptotic eccentricity invariant at the quadrupolar order, introduced in Ref. [48, and extended to $2 \mathrm{PN}$ in Ref. [4]. In fact, we adapted the approach of Ref. [49] by employing the appropriately modified 3PNaccurate $d \omega / d t$ and $d e_{t} / d t$ expressions of Refs. [50, 51. to obtain $3 \mathrm{PN}$-accurate bivariate expression for $e_{t}$. $\mathrm{A}$ careful synthesis of the above listed PN-accurate expressions lead to a fully analytic frequency domain TaylorF2 approximant that included $1 \mathrm{PN}$-accurate amplitude corrections and $3 \mathrm{PN}$-accurate Fourier phases. An additional feature of our approximant is the inclusion of periastron advance effects to $3 \mathrm{PN}$ order. To explore GW data analysis implications of these features, we perform preliminary match computations 52. We conclude that the influences of periastron advance are non-negligible for moderately eccentric binaries, especially in the aLIGO frequency window. This observation should be relevant while constructing IMR waveform family for compact binaries merging along moderate eccentric orbits.

This paper is structured as follows. In Sec. II. we summarize the efforts of Refs. 44, 49, to obtain analytic $\tilde{h}(f)$ with $\mathrm{PN}$-accurate Fourier phase. The crucial inputs to construct our eccentric TaylorF2 approximant is also listed in this section. Our approach and crucial expressions to implement our eccentric approximant that incorporates eccentricity contributions up to $\mathcal{O}\left(e_{t}^{6}\right)$ to $3 \mathrm{PN}$ are presented in Sec. III. A brief summary and possible extensions are listed in Sec. IV] while detailed expressions, accurate to $\mathcal{O}\left(e_{0}^{4}\right)$ are given in Appendix $\mathrm{C}$ 


\section{POST-CIRCULAR EXTENSIONS TO CIRCULAR INSPIRAL TEMPLATES}

We begin by reviewing two key efforts to include the effects of orbital eccentricity onto the circular inspiral templates [44, 48. This involves listing in Sec. II A the steps that are crucial to compute analytic frequency domain GW response function with quadrupolar amplitudes and PN-accurate Fourier phase in some detail. Various lengthy expressions, extracted from Refs. 45, 50, 51, are listed in Sec. IIB that will be crucial to compute the time domain response function for eccentric binaries while incorporating effects of periastron advance, higher order radiation reaction and amplitude corrections.

\section{A. Quadrupolar order $\tilde{h}(f)$ with PN-accurate Fourier phase}

Following 53, we may express the GW interferometric response function as

$$
h(t)=F_{+}\left(\theta_{S}, \phi_{S}, \psi_{S}\right) h_{+}(t)+F_{\times}\left(\theta_{S}, \phi_{S}, \psi_{S}\right) h_{\times}(t),
$$

where $F_{\times,+}\left(\theta_{S}, \phi_{S}, \psi_{S}\right)$ are the two detector antenna patterns. These quantities depend on $\phi_{S}, \theta_{S}$, the right ascension and declination of the source, and certain polarization angle $\psi_{S}$ 53. For eccentric inspirals, the explicit expressions for the quadrupolar order GW polarization states, $h_{\times}$and $h_{+}$, are given by Eqs. (3.1) of Ref. [44]. It is rather straightforward to express these Newtonian accurate expressions as a sum over harmonics in terms of the mean anomaly $l$. The resulting expressions read

$$
h_{+, \times}(t)=-\frac{G m \eta}{c^{2} D_{L}} x \sum_{j=1}^{10}\left[C_{+, \times}^{(j)} \cos j l+S_{+, \times}^{(j)} \sin j l\right]
$$

where $D_{L}$ denotes the luminosity distance while the symmetric mass ratio $\eta$ of a binary consisting of individual masses $m_{1}$ and $m_{2}$ is defined to be $\eta=\left(m_{1} m_{2}\right) / m^{2}$ while the total mass $m=m_{1}+m_{2}$. Further, we use the commonly used dimensionless PN expansion parameter $x=\left(\frac{G m \omega}{c^{3}}\right)^{2 / 3}$ where $G, c$ and $\omega$ are the gravitational constant, the speed of light in vacuum and the orbital angular frequency, respectively. The Newtonian accurate amplitudes, $C_{+, \times}^{(j)}$ and $S_{+, \times}^{(j)}$, are written as power series in orbital eccentricity $e_{t}$ whose coefficients involve trigonometric functions of the two angles $\iota, \beta$ that specify the line of sight vector in a certain inertial frame. The derivation of these expressions is detailed in Ref. [44] and the required inputs are obtained by adapting a standard analytic approach to solve the classical Kepler equation in terms of the Bessel functions [54].
With the help of Eqs. 2.1 and 2.2), we obtain interferometric strain for $\mathrm{GW}$ from eccentric binaries as

$$
h(t)=-\frac{G m \eta}{c^{2} D_{L}}\left(\frac{G m \omega}{c^{3}}\right)^{2 / 3} \sum_{j=1}^{10} \alpha_{j} \cos \left(j l+\phi_{j}\right)
$$

where $\alpha_{j}=\operatorname{sign}\left(\Gamma_{j}\right) \sqrt{\Gamma_{j}^{2}+\Sigma_{j}^{2}}$ and $\phi_{j}=\tan ^{-1}\left(-\frac{\Sigma_{j}}{\Gamma_{j}}\right)$. The two new functions, $\Gamma_{j}$ and $\Sigma_{j}$, are defined as $\Gamma_{j}=$ $F_{+} C_{+}^{(j)}+F_{\times} C_{\times}^{(j)}$ and $\Sigma_{j}=F_{+} S_{+}^{(j)}+F_{\times} S_{\times}^{(j)}$, respectively as in Ref. [4]. We impose the effects of GW emission on the above strain by specifying how $e_{t}$ and $\omega=2 \pi F, F$ being the orbital frequency, vary in time. In Ref. 44, the temporal evolutions of $\omega$ and $e_{t}$ are governed by the following Newtonian (or quadrupolar) equations that were adapted from Refs. 55 57.

$$
\begin{aligned}
\frac{d \omega}{d t} & =\frac{(G m \omega)^{5 / 3} \omega^{2} \eta}{5 c^{5}\left(1-e_{t}^{2}\right)^{7 / 2}}\left\{96+292 e_{t}^{2}+37 e_{t}{ }^{4}\right\}, \\
\frac{d e_{t}}{d t} & =-\frac{(G m \omega)^{5 / 3} \omega \eta e_{t}}{15 c^{5}\left(1-e_{t}^{2}\right)^{5 / 2}}\left\{304+121 e_{t}^{2}\right\} .
\end{aligned}
$$

It is customary to solve these two coupled differential 
temporally evolving $h(t)$. Interestingly, earlier efforts provided certain analytic way for obtaining temporal evolution for $\omega(t)$ and $e_{t}(t)$ that mainly involves the usage of hypergeometric functions [58 61]

However, it is possible to obtain analytic frequency domain counterpart of the above $h(t)$ as demonstrated in Ref. 44, 48. This traditional approach involves the method of SPA, detailed in Ref. 62, to compute analytically the Fourier Transform of $h(t)$. This was essentially demonstrated at the leading order in initial eccentricity $e_{0}$ in Ref. [48 and later extended to $\mathcal{O}\left(e_{0}^{8}\right)$ in Ref. [44]. Following Refs. 44, 48, we write

$$
\tilde{h}(f)=\tilde{\mathcal{A}}\left(\frac{G m \pi f}{c^{3}}\right)^{-7 / 6} \sum_{j=1}^{10} \xi_{j}\left(\frac{j}{2}\right)^{2 / 3} e^{-i\left(\pi / 4+\Psi_{j}\right)},
$$

where the overall amplitude $\tilde{\mathcal{A}}$ and the amplitudes of Fourier coefficients $\xi_{j}$ are given by

$$
\begin{aligned}
\tilde{\mathcal{A}} & =-\left(\frac{5 \eta \pi}{384}\right)^{1 / 2} \frac{G^{2} m^{2}}{c^{5} D_{L}} \\
\xi_{j} & =\frac{\left(1-e_{t}^{2}\right)^{7 / 4}}{\left(1+\frac{73}{24} e_{t}^{2}+\frac{37}{96} e_{t}^{4}\right)^{1 / 2}} \alpha_{j} e^{-i \phi_{j}(f / j)} .
\end{aligned}
$$

In the approach of stationary phase approximation, the crucial Fourier phase is given by

$$
\Psi_{j}\left[F\left(t_{0}\right)\right]=2 \pi \int^{F\left(t_{0}\right)} \tau^{\prime}\left(j-\frac{f}{F^{\prime}}\right) d F^{\prime}
$$

where $\tau$ stands for $F / \dot{F}$. Note that one needs to evaluate the above integrals at appropriate stationary points $t_{0}$, defined by $F\left(t_{0}\right)=f / j$.
To obtain a fully analytic ready-to-use expression for $\tilde{h}(f)$, we need to follow few additional steps. Clearly, we require to specify the frequency evolution of $e_{t}$ with the help of above Eqs. 2.4. The structure of these equations for $\dot{\omega}$ and $\dot{e}_{t}$ allows us to write $d \omega / d e_{t}=\omega \kappa_{N}\left(e_{t}\right)$ and it turns out that $\kappa_{N}$ depends only on $e_{t}$. This allows to integrate analytically the resulting $d \omega / \omega=\kappa_{N}\left(e_{t}\right) d e_{t}$ equation. The resulting expression can be written symbolically as $\omega / \omega_{0}=\kappa^{\prime}\left(e_{t}, e_{0}\right)$ where $e_{0}$ is the value of $e_{t}$ at the initial $\omega$ value, namely $\omega_{0}$ (see Eq. (62) in Ref. 34] for the explicit form for $\left.\kappa^{\prime}\left(e_{t}, e_{0}\right)\right)$. Interestingly, one may invert such an expression in the limit $e_{t} \ll 1$ to obtain $e_{t}$ in terms of $e_{0}, \omega$ and $\omega_{0}$ and it reads

$$
e_{t} \sim e_{0} \chi^{-19 / 18}+\mathcal{O}\left(e_{0}^{3}\right),
$$

where $\chi$ is defined as $\omega / \omega_{0}=F / F_{0}$. We note that the above result was first obtained in Ref. [48, which influenced them to introduce the idea of an asymptotic eccentric invariant. This relation allows us to write $\tau$ in terms of $\omega, \omega_{0}$ and $e_{0}$ as

$$
\tau \sim \frac{5}{96 \eta x^{4}}\left(\frac{G m}{c^{3}}\right)\left[1-\frac{157 e_{0}^{2}}{24} \chi^{-19 / 9}+\mathcal{O}\left(e_{0}^{4}\right)\right] .
$$

It is now straightforward to compute analytically the indefinite integral for $\Psi_{j}$, namely

$$
2 \pi \int \tau^{\prime}\left(j-\frac{f}{F^{\prime}}\right) d F^{\prime}
$$

that appears in Eq. 2.7 for $\tilde{h}(f)$. This leads to the following expression for $\Psi_{j}$, accurate to $\mathcal{O}\left(e_{0}^{2}\right)$ corrections:

$$
\Psi_{j} \sim j \phi_{c}-2 \pi f t_{c}-\frac{3}{128 \eta}\left(\frac{G m \pi f}{c^{3}}\right)^{-5 / 3}\left(\frac{j}{2}\right)^{8 / 3}\left[1-\frac{2355 e_{0}^{2}}{1462} \chi^{-19 / 9}+\mathcal{O}\left(e_{0}^{4}\right)\right]
$$


stands for $f / f_{0}$ due to the use of the stationary phase condition. Additionally, we have re-scaled $F_{0} \rightarrow f_{0} / j$ to ensure that $e_{t}\left(f_{0}\right)=e_{0}$ while employing the above expression for $e_{t}$, given by Eq. 2.8). Indeed, our expression is consistent with Eq. (4.28) of Ref. 44 that employs the chirp mass to characterize the binary. A number of extensions to the above result is available in the literature. In fact, Ref. 44 computed the higher order corrections to $e_{t}$ in terms of $e_{0}$ up to $\mathcal{O}\left(e_{0}^{7}\right)$ and extended $\Psi_{j}$ to $\mathcal{O}\left(e_{0}^{8}\right)$. Its PN extension, available in Ref. [49, provided 2PN corrections for $\Psi_{j}$ that incorporated eccentricity corrections, accurate to $\mathcal{O}\left(e_{0}^{6}\right)$ at every PN order while Ref. 63. computed $3 \mathrm{PN}$-accurate $\Psi_{j}$ that included leading order $e_{0}$ contributions.

A crucial ingredient to such $\mathrm{PN}$ extensions is the derivation of PN-accurate $e_{t}$ expression in terms of $e_{0}, \chi$ and $x$. In what follows, we summarize the steps that are required to obtain $1 \mathrm{PN}$-accurate expression for $e_{t}$ (see Ref. [49] for details). The starting point of such a derivation is the $1 \mathrm{PN}$-accurate differential equations for $\omega$ and $e_{t}$, obtainable from Eqs. (3.12) in Ref. [49. With these inputs, it is fairly straightforward to obtain the following $1 \mathrm{PN}$ accurate expression for $d \omega / \omega$ that includes only the leading order $e_{t}$ contributions as

$$
d \omega / \omega=\left\{-\frac{18}{19 e_{t}}-\frac{3}{10108 e_{t}}(-2833+5516 \eta)\left(\frac{G m \omega}{c^{3}}\right)^{2 / 3}\right\} d e_{t} .
$$

The fact that $\omega$ term appears only at the $1 \mathrm{PN}$ order allows us to use the earlier derived Newtonian accurate $\omega=\omega_{0}\left(e_{0} / e_{t}\right)^{18 / 19}$ relation to replace $\omega$ on the right hand side of the above equation. This leads to

$$
d \omega / \omega \sim\left\{-\frac{18}{19 e_{t}}-\frac{3}{10108}\left(\frac{e_{0}^{12 / 19}}{e_{t}^{31 / 19}}\right)(-2833+5516 \eta) x_{0}\right\} d e_{t},
$$

where $x_{0}=\left(G m \omega_{0} / c^{3}\right)^{2 / 3}$. We can integrate this equa-

tion to obtain $\ln \omega-\ln \omega_{0}$ in terms of $e_{t}, e_{0}$ and $\omega_{0}$. The exponential of the resulting expression and its bivariate expansion in terms of $x_{0}$ and $e_{t}$ result in

$$
\omega \sim\left\{\left(\frac{e_{0}}{e_{t}}\right)^{18 / 19}+x_{0}\left(\frac{2833-5516}{2128} \eta\right)\left[\left(\frac{e_{0}}{e_{t}}\right)^{18 / 19}-\left(\frac{e_{0}}{e_{t}}\right)^{30 / 19}\right]\right\} \omega_{0}
$$

We invert the above equation to obtain $e_{t}$ in terms of $e_{0}$ and $x_{0}$ after invoking the Newtonian accurate relation $e_{t}=e_{0} \chi^{-19 / 18}$ to replace the $e_{t}$ terms associated with the $x_{0}$ term. This inversion and the associated bivariate expansion in terms of $e_{0}$ and $x_{0}$ require that $e_{0} \ll 1$ and $x_{0} \ll 1$. The resulting $e_{t}$ expression reads

$$
e_{t} \sim e_{0}\left\{\chi^{-19 / 18}+x_{0}\left(\frac{2833}{2016}-\frac{197}{72} \eta\right)\left(-\chi^{-7 / 18}+\chi^{-19 / 18}\right)\right\}
$$

To obtain $e_{t}$ as a bivariate expansion in terms of the regular PN parameter $x$ and $e_{0}$, we employ the fact that $x / x_{0}=\chi^{2 / 3}$ and this results in 


$$
e_{t} \sim e_{0}\left\{\chi^{-19 / 18}+x\left(\frac{2833}{2016}-\frac{197}{72} \eta\right)\left(-\chi^{-19 / 18}+\chi^{-31 / 18}\right)\right\}
$$

We are now in a position to obtain $1 \mathrm{PN}$-accurate $\Psi_{j}$ expression that includes $\mathcal{O}\left(e_{0}^{2}\right)$ contributions both at the Newtonian and 1PN orders with the help of $1 \mathrm{PN}$-accurate $\tau=\omega / \dot{\omega}$ expression that is accurate to $\mathcal{O}\left(e_{t}^{2}\right)$ terms. A straightforward computation leads to the desired $\Psi_{j}$ expression which reads

$$
\begin{aligned}
\Psi_{j} \sim & j \phi_{c}-2 \pi f t_{c}-\left(\frac{3 j}{256 \eta}\right) x^{-5 / 2}\left\{1-\frac{2355 e_{0}^{2}}{1462} \chi^{-19 / 9}+x\left[\frac{3715}{756}+\frac{55}{9} \eta+\left(\left[-\frac{2045665}{348096}-\frac{128365}{12432} \eta\right] \chi^{-19 / 9}\right.\right.\right. \\
+ & {\left.\left.\left.\left[-\frac{2223905}{491232}+\frac{154645}{17544} \eta\right] \chi^{-25 / 9}\right) e_{0}^{2}\right]\right\} }
\end{aligned}
$$

where the quantities $x$ and $\chi$ will have to be evaluated at the stationary point (see Ref. [49] for details). With the above equation, we explicitly listed our approach to compute $\mathrm{PN}$-accurate $\Psi_{j}$ that incorporates $e_{0}$ corrections at each PN order. In the present paper, we extend these computations to $3 \mathrm{PN}$ order while incorporating $\mathcal{O}\left(e_{0}^{6}\right)$ contributions at each PN order. These higher order $e_{0}$ corrections are included as we desire to to model GWs from moderately eccentric compact binary inspirals. In the next section, we provide crucial inputs that will be required to compute analytic $1 \mathrm{PN}$-accurate amplitude corrected $\tilde{h}(f)$ with $3 \mathrm{PN}$-accurate Fourier phases.

\section{B. Analytic PN-accurate amplitude corrected time domain eccentric GW templates}

The previous section showed that we require analytic expressions for the two GW polarization states as a sum over harmonics to construct ready-to-use analytic $\tilde{h}(f)$. This influenced us to adapt Eqs. (44) and (45) in Ref. 45] that provided analytic 1PN-accurate amplitude corrected $h_{\times,+}(t)$ which additionally included the effects of periastron advance on individual harmonics. This may be seen by a close inspection of appropriate terms in Eqs. (44),(45),(46) and (47) of Ref. [45. To describe in detail how these improvements in GW polarization states change the harmonic structure of $h(t)$, we restrict our attention to quadrupolar order contributions to $h_{\times,+}(t)$, given in Eqs. (44) and (45) of Ref. [45. The explicit expressions for such 'Newtonian' contributions to $h_{\times,+}(t)$ that include $\mathcal{O}\left(e_{t}^{4}\right)$ corrections read

$$
\begin{aligned}
h_{\times}^{0}= & \frac{G m \eta}{c^{2} D_{L}} x\left\{\cos \left(\phi+\phi^{\prime}\right)\left[\left(-3 e_{t}+\frac{13 e_{t}^{3}}{8}\right) c_{i} s_{2 \beta}\right]+\sin \left(\phi+\phi^{\prime}\right)\left[\left(3 e_{t}-\frac{13 e_{t}^{3}}{8}\right) c_{i} c_{2 \beta}\right]+\cos (2 \phi)\left[\left(4-10 e_{t}^{2}\right.\right.\right. \\
& \left.\left.+\frac{23 e_{t}^{4}}{4}\right) c_{i} s_{2 \beta}\right]+\sin (2 \phi)\left[\left(-4+10 e_{t}^{2}-\frac{23 e_{t}^{4}}{4}\right) c_{i} c_{2 \beta}\right]+\cos \left(3 \phi-\phi^{\prime}\right)\left[\left(9 e_{t}-\frac{171 e_{t}^{3}}{8}\right) c_{i} s_{2 \beta}\right] \\
& +\sin \left(3 \phi-\phi^{\prime}\right)\left[\left(-9 e_{t}+\frac{171 e_{t}^{3}}{8}\right) c_{i} c_{2 \beta}\right]+\cos \left(4 \phi-2 \phi^{\prime}\right)\left[\left(16 e_{t}^{2}-40 e_{t}^{4}\right) c_{i} s_{2 \beta}\right]+\sin \left(4 \phi-2 \phi^{\prime}\right)\left[\left(-16 e_{t}^{2}\right.\right. \\
& \left.\left.+40 e_{t}^{4}\right) c_{i} c_{2 \beta}\right]+\cos \left(5 \phi-3 \phi^{\prime}\right)\left[\frac{625}{24} e_{t}^{3} c_{i} s_{2 \beta}\right]+\sin \left(5 \phi-3 \phi^{\prime}\right)\left[\frac{-625}{24} e_{t}^{3} c_{i} c_{2 \beta}\right]+\cos \left(6 \phi-4 \phi^{\prime}\right)\left[\frac{81}{2} e_{t}^{4} c_{i} s_{2 \beta}\right] \\
& +\sin \left(6 \phi-4 \phi^{\prime}\right)\left[\frac{-81}{2} e_{t}^{4} c_{i} c_{2 \beta}\right]+\cos \left(\phi-3 \phi^{\prime}\right)\left[\frac{-7}{24} e_{t}^{3} c_{i} s_{2 \beta}\right]+\sin \left(\phi-3 \phi^{\prime}\right)\left[\frac{-7}{24} e_{t}^{3} c_{i} c_{2 \beta}\right] \\
& \left.+\cos \left(2 \phi-4 \phi^{\prime}\right)\left[-\frac{1}{4} e_{t}^{4} c_{i} s_{2 \beta}\right]+\sin \left(2 \phi-4 \phi^{\prime}\right)\left[-\frac{1}{4} e_{t}^{4} c_{i} c_{2 \beta}\right]\right\}
\end{aligned}
$$




$$
\begin{aligned}
h_{+}^{0}= & \frac{G m \eta}{c^{2} D_{L}} x\left\{\cos \left(\phi+\phi^{\prime}\right)\left[\left(\frac{3 e_{t}}{2}-\frac{13 e_{t}^{3}}{16}\right)\left(1+c_{i}^{2}\right) c_{2 \beta}\right]+\sin \left(\phi+\phi^{\prime}\right)\left[\left(\frac{3 e_{t}}{2}-\frac{13 e_{t}^{3}}{16}\right)\left(1+c_{i}^{2}\right) s_{2 \beta}\right]\right. \\
& +\cos (2 \phi)\left[\left(-2+5 e_{t}^{2}-\frac{23 e_{t}^{4}}{8}\right)\left(1+c_{i}^{2}\right) c_{2 \beta}\right]+\sin (2 \phi)\left[\left(-2+5 e_{t}^{2}-\frac{23 e_{t}^{4}}{8}\right)\left(1+c_{i}^{2}\right) s_{2 \beta}\right] \\
& +\cos \left(3 \phi-\phi^{\prime}\right)\left[\left(-\frac{9 e_{t}}{2}+\frac{171 e_{t}^{3}}{16}\right)\left(1+c_{i}^{2}\right) c_{2 \beta}\right]+\sin \left(3 \phi-\phi^{\prime}\right)\left[\left(-\frac{9 e_{t}}{2}+\frac{171 e_{t}^{3}}{16}\right)\left(1+c_{i}^{2}\right) s_{2 \beta}\right] \\
& +\cos \left(4 \phi-2 \phi^{\prime}\right)\left[\left(-8 e_{t}^{2}+20 e_{t}^{4}\right)\left(1+c_{i}^{2}\right) c_{2 \beta}\right]+\sin \left(4 \phi-2 \phi^{\prime}\right)\left[\left(-8 e_{t}^{2}+20 e_{t}^{4}\right)\left(1+c_{i}^{2}\right) s_{2 \beta}\right] \\
& +\cos \left(5 \phi-3 \phi^{\prime}\right)\left[-\frac{625}{48} e_{t}^{3}\left(1+c_{i}^{2}\right) c_{2 \beta}\right]+\sin \left(5 \phi-3 \phi^{\prime}\right)\left[-\frac{625}{48} e_{t}^{3}\left(1+c_{i}^{2}\right) s_{2 \beta}\right]+\cos \left(6 \phi-4 \phi^{\prime}\right)\left[-\frac{81}{4} e_{t}^{4}\left(1+c_{i}^{2}\right) c_{2 \beta}\right] \\
& +\sin \left(6 \phi-4 \phi^{\prime}\right)\left[-\frac{81}{4} e_{t}^{4}\left(1+c_{i}^{2}\right) s_{2 \beta}\right]+\cos \left(\phi-\phi^{\prime}\right)\left[\left(e_{t}-\frac{e_{t}^{3}}{8}\right) s_{i}^{2}\right]+\cos \left(2 \phi-2 \phi^{\prime}\right)\left[\left(e_{t}^{2}-\frac{e_{t}^{4}}{3}\right) s_{i}^{2}\right] \\
& +\cos \left(3 \phi-3 \phi^{\prime}\right)\left[\frac{9}{8} e_{t}^{3} s_{i}^{2}\right]+\cos \left(4 \phi-4 \phi^{\prime}\right)\left[\frac{4}{3} e_{t}^{4} s_{i}^{2}\right]+\cos \left(\phi-3 \phi^{\prime}\right)\left[\frac{7}{48} e_{t}^{3}\left(1+c_{i}^{2}\right) c_{2 \beta}\right] \\
& \left.+\sin \left(\phi-3 \phi^{\prime}\right)\left[-\frac{7}{48} e_{t}^{3}\left(1+c_{i}^{2}\right) s_{2 \beta}\right]+\cos \left(2 \phi-4 \phi^{\prime}\right)\left[-\frac{1}{8} e_{t}^{4}\left(1+c_{i}^{2}\right) c_{2 \beta}\right]+\sin \left(2 \phi-4 \phi^{\prime}\right)\left[-\frac{1}{8} e_{t}^{4}\left(1+c_{i}^{2}\right) s_{2 \beta}\right]\right\}
\end{aligned}
$$

where $\phi=(1+k) l, \phi^{\prime}=k l$ and $k$ provides the rate of periastron advance per orbit [34]. Further, we let $c_{i}=\cos \iota$, $s_{i}=\sin \iota, c_{2 \beta}=\cos 2 \beta$ and $s_{2 \beta}=\sin 2 \beta$. Note that crucial ingredients to obtain above analytic expressions include developing approaches to solve PN-accurate Kepler equation and adapting them to derive $\mathrm{PN}$-accurate relations to connect true and eccentric anomalies, detailed in Ref. 45]. A close inspection of the above two equations with Eqs. (3.3) and (3.4) of Ref. 44] reveals that the arguments of cosine and sine functions in above expressions involve $\phi^{\prime}=k l$ and its multiples in addition to the usual orbital phase $\phi$ and its multiples. These additional $\phi^{\prime}$ contributions are clearly due to the perias- tron advance. It turns out that these additional angular contributions are sufficient to provide the numerically inferred side bands in the power spectrum of eccentric binaries due to the presence of $k$ [46. This is why we explicitly included $e_{t}^{4}$ contributions to the above $h_{\times,+}$ expressions as these contributions are required to reveal the underlying side band structure of waveforms due to the influence of periastron advance.

We re-write the above expressions for $h_{\times,+}^{0}$ in a more compact form to explicitly show how various harmonics are affected by the advance of periastron. The resulting expressions read

$$
\begin{aligned}
h_{+, \times}^{0}(t)= & \left\{\sum_{j=1}^{6}\left[C_{+, \times}^{j,-2}(0) \cos \left(j \phi-(j-2) \phi^{\prime}\right)+S_{+, \times}^{j,-2}(0) \sin \left(j \phi-(j-2) \phi^{\prime}\right)\right]+\sum_{j=1}^{4}\left[C_{+, \times}^{j, 0}(0) \cos \left(j \phi-j \phi^{\prime}\right)\right.\right. \\
& \left.\left.+S_{+, \times}^{j, 0}(0) \sin \left(j \phi-j \phi^{\prime}\right)\right]+\sum_{j=1}^{2}\left[C_{+, \times}^{j,+2}(0) \cos \left(j \phi-(j+2) \phi^{\prime}\right)+S_{+, \times}^{j,+2}(0) \sin \left(j \phi-(j+2) \phi^{\prime}\right)\right]\right\},
\end{aligned}
$$

where we denoted the coefficient of $\cos \left(j \phi-(j \pm n) \phi^{\prime}\right)$ harmonic at the quadrupolar (Newtonian) order for the + polarization by $C_{+}^{j, \pm n}(0)$ while the coefficient of $\sin (j \phi-$ $\left.(j \pm n) \phi^{\prime}\right)$ is indicated by $S_{+}^{j, \pm n}(0)$. We adopt a rather heavy notation as it is amenable to higher PN order contributions which will be tackled below. In this convention, we represent the coefficient of $\cos \left(j \phi-(j \pm n) \phi^{\prime}\right)$ that appears in the $1 \mathrm{PN}$ contributions to $\times$ polarization state by $C_{\times}^{j, \pm n}(1)$. It should be obvious that $j$ stands for the harmonic variable while $n$ provides a measure of the shift that each harmonic experiences due to periastron advance. A close comparison of Eqs. 2.18) and (2.19) reveals that these coefficients are functions of $\iota, \beta$ and contain powers of $e_{t}$. Moreover, the arguments of cosine and sine functions clearly show that the eccentricity induced higher harmonics are not mere multiples of $\omega=N(1+k)$, where $N$ is the PN-accurate mean motion. Clearly, this is due to the presence of non-vanishing $\phi^{\prime}$ contributions due to periastron advance. Interestingly, the plus polar- 
ization state does provide harmonics which are integer multiples of $N$. It is not difficult to show that these Newtonian like terms arise from specific cosine functions with arguments $j \phi-j \phi^{\prime}$, as evident from Eqs. 2.19. Further, it is possible to show that these contributions arise from $e_{t} \cos u s_{i}^{2} /\left(1-e_{t} \cos u\right)$ contributions to $H_{+}^{0}$, given by Eq. (F2a) in Ref. 45, and therefore not influenced by the periastron advance. Interestingly, similar conclusions were obtained in Ref. 46.

With the above inputs, we write the time-domain GW detector response function for eccentric inspirals as

$$
\begin{aligned}
h(t)= & \frac{G m \eta}{c^{2} D_{L}} x\left\{\sum_{j=1}^{6}\left[\Gamma_{j,-2}^{(0)} \cos \left(j \phi-(j-2) \phi^{\prime}\right)+\Sigma_{j,-2}^{(0)} \sin \left(j \phi-(j-2) \phi^{\prime}\right)\right]+\sum_{j=1}^{4}\left[\Gamma_{j, 0}^{(0)} \cos \left(j \phi-j \phi^{\prime}\right)\right.\right. \\
& \left.\left.+\Sigma_{j, 0}^{(0)} \sin \left(j \phi-j \phi^{\prime}\right)\right]+\sum_{j=1}^{2}\left[\Gamma_{j,+2}^{(0)} \cos \left(j \phi-(j+2) \phi^{\prime}\right)+\Sigma_{j,+2}^{(0)} \sin \left(j \phi-(j+2) \phi^{\prime}\right)\right]\right\},
\end{aligned}
$$

where the amplitudes of the cosine and sine functions are denoted by rather complicated symbols $\Gamma_{j, \pm n}^{(0)}$ and $\Sigma_{j, \pm n}^{(0)}$. The definition of $h(t)=F_{+} h_{+}(t)+F_{\times} h_{\times}(t)$ ensures that $\Gamma_{j, \pm n}^{(0)}=F_{+} C_{+}^{j, \pm n}(0)+F_{\times} C_{\times}^{j, \pm n}(0)$ while $\Sigma_{j, \pm n}^{(0)}=F_{+} S_{+}^{j, \pm n}(0)+F_{\times} S_{\times}^{j, \pm n}(0)$. We list in Appendix $\mathrm{A}$, the lengthy expressions for these quantities in terms of $\iota, \beta$ and eccentricity contributions, accurate to $\mathcal{O}\left(e_{t}^{4}\right)$. We display up to $\mathcal{O}\left(e_{t}^{4}\right)$ contributions to demonstrate the full harmonic structure of the quadrupolar order GW polarization states. It turns out that $\Sigma_{j, 0}^{(0)}$ contributions are zero by construction. This is mainly because the un-shifted harmonics only appear with the cosine terms, present in the + polarization state. Invoking familiar trigonometric identities, we simplify the above equation and obtain

$$
\begin{aligned}
h(t)= & \frac{G m \eta}{c^{2} D_{L}} x\left\{\sum_{j=1}^{6} \alpha_{j,-2}^{(0)} \cos \left(j \phi-(j-2) \phi^{\prime}+\bar{\phi}_{j,-2}^{(0)}\right)+\sum_{j=1}^{4} \alpha_{j, 0}^{(0)} \cos \left(j \phi-j \phi^{\prime}+\bar{\phi}_{j, 0}^{(0)}\right)\right. \\
& \left.+\sum_{j=1}^{2} \alpha_{j,+2}^{(0)} \cos \left(j \phi-(j+2) \phi^{\prime}+\bar{\phi}_{j,+2}^{(0)}\right)\right\},
\end{aligned}
$$

where we introduce two new multi-index symbols $\alpha_{j, \pm n}^{(0)}$ and $\bar{\phi}_{j, \pm n}^{(0)}$ to ensure that detector strain can be written in terms of only cosine functions. Influenced by Ref. 44, these symbols are defined as $\alpha_{j, \pm n}^{(0)}=\operatorname{sign}\left(\Gamma_{j, \pm n}^{(0)}\right) \sqrt{\left(\Gamma_{j, \pm n}^{(0)}\right)^{2}+\left(\Sigma_{j, \pm n}^{(0)}\right)^{2}}$ and $\bar{\phi}_{j, \pm n}^{(0)}=\tan ^{-1}\left(-\frac{\Sigma_{j, \pm n}^{(0)}}{\Gamma_{j, \pm n}^{(0)}}\right)$. We do not list explicit expressions for these quantities that are accurate to $\mathcal{O}\left(e_{t}^{4}\right)$ in eccentricity corrections as they can be easily obtained from our Eqs. A1 and A2.

A close inspection of above equations reveal that they provide GW response function for compact binaries mov- ing along precessing eccentric orbits. To obtain temporally evolving $h(t)$ associated with compact binaries inspiraling along precessing eccentric orbits, we need to specify how $\phi, \phi^{\prime}, \omega$ and $e_{t}$ vary in time due to GW emission. We adapt the phasing formalism, detailed in Refs. [34, 49, to provide differential equations for these variables. And, for the time being, we will concentrate on the secular evolution of these variables. In other words, we will neglect GW induced quasi-periodic variations to orbital elements and angles, detailed in Ref. 34. The $3 \mathrm{PN}$-accurate secular evolution to $\phi$ and $\phi^{\prime}$ in the modified harmonic gauge that are accurate to $\mathcal{O}\left(e_{t}^{6}\right)$ are given by

$$
\frac{d \phi}{d t}=\omega=x^{3 / 2} \frac{c^{3}}{G m}
$$




$$
\begin{aligned}
\frac{d \phi^{\prime}}{d t}= & \omega \frac{k}{1+k}=\omega\left\{3 x\left[1+e_{t}^{2}+e_{t}^{4}+e_{t}^{6}\right]+x^{2}\left[\frac{9}{2}-7 \eta+\left(\frac{87}{4}-\frac{41}{2} \eta\right) e_{t}^{2}+(39-34 \eta) e_{t}^{4}+\left(\frac{225}{4}-\frac{95}{2} \eta\right) e_{t}^{6}\right]\right. \\
& +x^{3}\left[\frac{27}{2}+\left(-\frac{481}{4}+\frac{123 \pi^{2}}{32}\right) \eta+7 \eta^{2}+\left(\frac{519}{4}+\left(-\frac{2037}{4}+\frac{1599 \pi^{2}}{128}\right) \eta+61 \eta^{2}\right) e_{t}^{2}+\left(\frac{2811}{8}\right.\right. \\
& \left.\left.\left.+\left(-1174+\frac{3321 \pi^{2}}{128}\right) \eta+\frac{1361}{8} \eta^{2}\right) e_{t}^{4}+\left(\frac{10779}{16}+\left(-\frac{16901}{8}+\frac{2829 \pi^{2}}{64}\right) \eta+\frac{2675}{8} \eta^{2}\right) e_{t}^{6}\right]\right\}, \\
\frac{d \omega}{d t}= & \frac{96 c^{6} \eta}{5 G^{2} m^{2}} x^{11 / 2}\left\{1+\frac{157}{24} e_{t}^{2}+\frac{605}{32} e_{t}^{4}+\frac{3815}{96} e_{t}^{6}+x\left[-\frac{743}{336}-\frac{11}{4} \eta+\left(\frac{713}{112}-\frac{673}{16} \eta\right) e_{t}^{2}+\left(\frac{52333}{672}-\frac{12415}{64} \eta\right) e_{t}^{4}\right.\right. \\
& \left.\left.+\left(\frac{13823}{48}-\frac{107765}{192} \eta\right) e_{t}^{6}\right]+\dot{\omega}^{1.5 P N}+\dot{\omega}^{2 P N}+\dot{\omega}^{2.5 P N}+\dot{\omega}^{3 P N}\right\}, \\
\frac{d e_{t}}{d t}= & -\frac{304 c^{3} \eta e_{t}}{15 G m} x^{4}\left\{1+\frac{881}{304} e_{t}^{2}+\frac{3265}{608} e_{t}^{4}+\frac{20195}{2432} e_{t}^{6}+x\left[-\frac{2817}{2128}-\frac{1021}{228} \eta+\left(\frac{40115}{4256}-\frac{51847}{1824} \eta\right) e_{t}^{2}\right.\right. \\
& \left.\left.+\left(\frac{87749}{2128}-\frac{298115}{3648} \eta\right) e_{t}^{4}+\left(\frac{121833}{1216}-\frac{2501905}{14592} \eta\right) e_{t}^{6}\right]+\dot{e}_{t}^{1.5 P N}+\dot{e}_{t}^{2 P N}+\dot{e}_{t}^{2.5 P N}+\dot{e}_{t}^{3 P N}\right\} .
\end{aligned}
$$

The explicit 1.5, 2, 2.5 and 3PN order contributions to $d \omega / d t$ and $d e_{t} / d t$ that incorporates all the $\mathcal{O}\left(e_{t}^{6}\right)$ corrections are provided in the Appendix $B$ The differential equations for $d \omega / d t$ and $d e_{t} / d t$ are extracted from expressions, available in Refs. [50, 51] and are in the modified harmonic gauge. These papers provided above 3PN accurate expressions as sum of certain 'instantaneous' and 'tail' contributions

$$
\begin{aligned}
\frac{d \omega}{d t} & =\left(\frac{d \omega}{d t}\right)_{\mathrm{inst}}+\left(\frac{d \omega}{d t}\right)_{\mathrm{tail}}, \\
\frac{d e_{t}}{d t} & =\left(\frac{d e_{t}}{d t}\right)_{\mathrm{inst}}+\left(\frac{d e_{t}}{d t}\right)_{\mathrm{tail}} .
\end{aligned}
$$

The 3PN-accurate instantaneous contributions that depend only on the binary dynamics at the usual retarded time while the hereditary contributions are sensitive to the binary dynamics at all epochs prior to the usual retarded time 64. The instantaneous contributions to $d \omega / d t$ are extracted from Eqs. (6.14),(6.15a),(6.15),(C6) and $(\mathrm{C} 7)$ of Ref. 50] while for $d e_{t} / d t$ such contributions originate from Eqs. (6.16),(6.19a),(6.19b),(C10) and (C11) in Ref. [50. It should be obvious that we have Taylor expanded these equations around $e_{t}=0$ to obtain eccentricity contributions accurate to $\mathcal{O}\left(e_{t}^{6}\right)$. The hereditary contributions to $d \omega / d t$ and $d e_{t} / d t$ are adapted from
Eqs. (6.24c) and (6.26) of Ref. [50] and they depend on a number of eccentricity enhancement functions. We employ such enhancement functions provided in Ref. [51] for our computations.

We now have all the inputs to obtain the restricted timedomain $h(t)$ to model GWs from non-spinning compact binaries inspiraling along precessing moderately eccentric orbits. To obtain such time domain templates, we numerically solve the above listed differential equations for $\omega, e_{t}, \phi$ and $\phi^{\prime}$ and impose their temporal evolution in the quadrupolar order GW response function, given by Eq. (2.22). We now move onto describe how we extend the quadrupolar order GW response function.

It should be obvious that we require a prescription to compute analytically $\mathrm{PN}$-accurate amplitude corrected GW polarization states to improve the above listed quadrupolar order GW response function. Therefore, we adapt $1 \mathrm{PN}$-accurate amplitude corrected and fully analytic expressions for $h_{\times,+}$, available in Ref. [45], to compute GW response function for eccentric inspirals that incorporates PN contributions even to its amplitudes. We list below certain ingredients that will be crucial to write down analytic $h(t)$ that incorporates $1 \mathrm{PN}$-accurate amplitude corrections to $h_{\times,+}$while consistently keeping eccentricity contributions up to $\mathcal{O}\left(e_{t}^{6}\right)$. We begin by displaying Eqs. (44) and (45) of Ref. [45] as a single sum which reads

$$
h_{+, \times}(t)=\frac{G m \eta}{c^{2} D_{L}} x\left\{h_{+, \times}^{0}(t)+x^{0.5} h_{+, \times}^{0.5}(t)+x h_{+, \times}^{1}(t)\right\} .
$$


Various PN order amplitude contributions take the following form

$$
\begin{aligned}
& h_{+, \times}^{0}(t)=\sum_{j=1}^{8}\left\{C_{+, \times}^{j,-2}(0) \cos \left(j \phi-(j-2) \phi^{\prime}\right)+S_{+, \times}^{j,-2}(0) \sin \left(j \phi-(j-2) \phi^{\prime}\right)\right\}+\sum_{j=1}^{6}\left\{C_{+, \times}^{j, 0}(0) \cos \left(j \phi-j \phi^{\prime}\right)\right. \\
& \left.+S_{+, \times}^{j, 0}(0) \sin \left(j \phi-j \phi^{\prime}\right)\right\}+\sum_{j=1}^{4}\left\{C_{+, \times}^{j,+2}(0) \cos \left(j \phi-(j+2) \phi^{\prime}\right)+S_{+, \times}^{j,+2}(0) \sin \left(j \phi-(j+2) \phi^{\prime}\right)\right\}, \\
& h_{+, \times}^{0.5}(t)=\delta\left\{\sum_{j=1}^{7}\left[C_{+, \times}^{j,-1}(0.5) \cos \left(j \phi-(j-1) \phi^{\prime}\right)+S_{+, \times}^{j,-1}(0.5) \sin \left(j \phi-(j-1) \phi^{\prime}\right)\right]+\sum_{j=1}^{5}\left[C_{+, \times}^{j,+1}(0.5) \cos \left(j \phi-(j+1) \phi^{\prime}\right)\right.\right. \\
& \left.+S_{+, \times}^{j,+1}(0.5) \sin \left(j \phi-(j+1) \phi^{\prime}\right)\right]+\sum_{j=1}^{9}\left[C_{+, \times}^{j,-3}(0.5) \cos \left(j \phi-(j-3) \phi^{\prime}\right)+S_{+, \times}^{j,-3}(0.5) \sin \left(j \phi-(j-3) \phi^{\prime}\right)\right] \\
& \left.+\sum_{j=1}^{3}\left[C_{+, \times}^{j,+3}(0.5) \cos \left(j \phi-(j+3) \phi^{\prime}\right)+S_{+, \times}^{j,+3}(0.5) \sin \left(j \phi-(j+3) \phi^{\prime}\right)\right]\right\} \\
& h_{+, \times}^{1}(t)=\sum_{j=1}^{8}\left\{C_{+, \times}^{j,-2}(1) \cos \left(j \phi-(j-2) \phi^{\prime}\right)+S_{+, \times}^{j,-2}(1) \sin \left(j \phi-(j-2) \phi^{\prime}\right)\right\}+\sum_{j=1}^{4}\left\{C_{+, \times}^{j,+2}(1) \cos \left(j \phi-(j+2) \phi^{\prime}\right)\right. \\
& \left.+S_{+, \times}^{j,+2}(1) \sin \left(j \phi-(j+2) \phi^{\prime}\right)\right\}+\sum_{j=1}^{10}\left\{C_{+, \times}^{j,-4}(1) \cos \left(j \phi-(j-4) \phi^{\prime}\right)+S_{+, \times}^{j,-4}(1) \sin \left(j \phi-(j-4) \phi^{\prime}\right)\right\} \\
& +\sum_{j=1}^{2}\left\{C_{+, \times}^{j,+4}(1) \cos \left(j \phi-(j+4) \phi^{\prime}\right)+S_{+, \times}^{j,+4}(1) \sin \left(j \phi-(j+4) \phi^{\prime}\right)\right\}+\sum_{j=1}^{6}\left\{C_{+, \times}^{j, 0}(1) \cos \left(j \phi-j \phi^{\prime}\right)\right. \\
& \left.+S_{+, \times}^{j, 0}(1) \sin \left(j \phi-j \phi^{\prime}\right)\right\}
\end{aligned}
$$

where $\delta=\left(m_{1}-m_{2}\right) /\left(m_{1}+m_{2}\right)$ and we let $m_{1}$ to be the heavier of the two binary components. We do not list explicitly very lengthy expressions for these amplitudes. However, they can be easily extracted from the attached Mathematica notebook. The derivation of above lengthy expressions include developing analytic approaches to solve $\mathrm{PN}$-accurate Kepler equation and $\mathrm{PN}$ - accurate relations connecting true and eccentric anomalies, detailed in Ref. 45. Indeed, we have verified that these expressions reduce to their circular counterparts, provided in Ref. 65.

The associated GW detector strain for eccentric binaries is given by

$$
\begin{aligned}
h(t)= & \frac{G m \eta}{c^{2} D_{L}} x\left\{\left[\sum_{j=1}^{8}\left(\Gamma_{j,-2}^{(0)} \cos \left(j \phi-(j-2) \phi^{\prime}\right)+\Sigma_{j,-2}^{(0)} \sin \left(j \phi-(j-2) \phi^{\prime}\right)\right)+\sum_{j=1}^{6}\left(\Gamma_{j, 0}^{(0)} \cos \left(j \phi-j \phi^{\prime}\right)+\Sigma_{j, 0}^{(0)} \sin \left(j \phi-j \phi^{\prime}\right)\right)\right.\right. \\
& \left.+\sum_{j=1}^{4}\left(\Gamma_{j,+2}^{(0)} \cos \left(j \phi-(j+2) \phi^{\prime}\right)+\Sigma_{j,+2}^{(0)} \sin \left(j \phi-(j+2) \phi^{\prime}\right)\right)\right]+x^{0.5} \delta\left[\sum _ { j = 1 } ^ { 7 } \left(\Gamma_{j,-1}^{(0.5)} \cos \left(j \phi-(j-1) \phi^{\prime}\right)\right.\right. \\
& \left.+\Sigma_{j,-1}^{(0.5)} \sin \left(j \phi-(j-1) \phi^{\prime}\right)\right)+\sum_{j=1}^{5}\left(\Gamma_{j,+1}^{(0.5)} \cos \left(j \phi-(j+1) \phi^{\prime}\right)+\Sigma_{j,+1}^{(0.5)} \sin \left(j \phi-(j+1) \phi^{\prime}\right)\right) \\
& +\sum_{j=1}^{9}\left(\Gamma_{j,-3}^{(0.5)} \cos \left(j \phi-(j-3) \phi^{\prime}\right)+\Sigma_{j,-3}^{(0.5)} \sin \left(j \phi-(j-3) \phi^{\prime}\right)\right)+\sum_{j=1}^{3}\left(\Gamma_{j,+3}^{(0.5)} \cos \left(j \phi-(j+3) \phi^{\prime}\right)\right. \\
& \left.\left.+\Sigma_{j,+3}^{(0.5)} \sin \left(j \phi-(j+3) \phi^{\prime}\right)\right)\right]+x\left[\sum_{j=1}^{8}\left(\Gamma_{j,-2}^{(1)} \cos \left(j \phi-(j-2) \phi^{\prime}\right)+\Sigma_{j,-2}^{(1)} \sin \left(j \phi-(j-2) \phi^{\prime}\right)\right)\right.
\end{aligned}
$$




$$
\begin{aligned}
& +\sum_{j=1}^{4}\left(\Gamma_{j,+2}^{(1)} \cos \left(j \phi-(j+2) \phi^{\prime}\right)+\Sigma_{j,+2}^{(1)} \sin \left(j \phi-(j+2) \phi^{\prime}\right)\right)+\sum_{j=1}^{6}\left(\Gamma_{j, 0}^{(1)} \cos \left(j \phi-j \phi^{\prime}\right)+\Sigma_{j, 0}^{(1)} \sin \left(j \phi-j \phi^{\prime}\right)\right) \\
& +\sum_{j=1}^{10}\left(\Gamma_{j,-4}^{(1)} \cos \left(j \phi-(j-4) \phi^{\prime}\right)+\Sigma_{j,-4}^{(1)} \sin \left(j \phi-(j-4) \phi^{\prime}\right)\right)+\sum_{j=1}^{2}\left(\Gamma_{j,+4}^{(1)} \cos \left(j \phi-(j+4) \phi^{\prime}\right)\right. \\
& \left.\left.\left.+\Sigma_{j,+4}^{(1)} \sin \left(j \phi-(j+4) \phi^{\prime}\right)\right)\right]\right\}
\end{aligned}
$$

where as expected, we have defined

$$
\begin{aligned}
& \Gamma_{j, \pm n}^{(p)}=F_{+} C_{+}^{j, \pm n}(p)+F_{\times} C_{\times}^{j, \pm n}(p) \\
& \Sigma_{j, \pm n}^{(p)}=F_{+} S_{+}^{j, \pm n}(p)+F_{\times} S_{\times}^{j, \pm n}(p)
\end{aligned}
$$

A further simplification is possible which requires, as expected, additional multi-index functions

$$
\alpha_{j, \pm n}^{(p)}=\operatorname{sign}\left(\Gamma_{j, \pm n}^{(p)}\right) \sqrt{\left(\Gamma_{j, \pm n}^{(p)}\right)^{2}+\left(\Sigma_{j, \pm n}^{(p)}\right)^{2}}
$$

such that

$$
\begin{aligned}
h(t)= & \frac{G m \eta}{c^{2} D_{L}} x\left\{\left[\sum_{j=1}^{8} \alpha_{j,-2}^{(0)} \cos \left(j \phi-(j-2) \phi^{\prime}+\bar{\phi}_{j,-2}^{(0)}\right)+\sum_{j=1}^{6} \alpha_{j, 0}^{(0)} \cos \left(j \phi-j \phi^{\prime}+\bar{\phi}_{j, 0}^{(0)}\right)\right.\right. \\
& \left.+\sum_{j=1}^{4} \alpha_{j,+2}^{(0)} \cos \left(j \phi-(j+2) \phi^{\prime}+\bar{\phi}_{j,+2}^{(0)}\right)\right]+x^{0.5} \delta\left[\sum_{j=1}^{7} \alpha_{j,-1}^{(0.5)} \cos \left(j \phi-(j-1) \phi^{\prime}+\bar{\phi}_{j,-1}^{(0.5)}\right)\right. \\
& +\sum_{j=1}^{5} \alpha_{j,+1}^{(0.5)} \cos \left(j \phi-(j+1) \phi^{\prime}+\bar{\phi}_{j,-1}^{(0.5)}\right)+\sum_{j=1}^{9} \alpha_{j,-3}^{(0.5)} \cos \left(j \phi-(j-3) \phi^{\prime}+\bar{\phi}_{j,-3}^{(0.5)}\right) \\
& \left.+\sum_{j=1}^{3} \alpha_{j,+3}^{(0.5)} \cos \left(j \phi-(j+3) \phi^{\prime}+\bar{\phi}_{j,+3}^{(0.5)}\right)\right]+x\left[\sum_{j=1}^{8} \alpha_{j,-2}^{(1)} \cos \left(j \phi-(j-2) \phi^{\prime}+\bar{\phi}_{j,-2}^{(1)}\right)\right. \\
& +\sum_{j=1}^{4} \alpha_{j,+2}^{(1)} \cos \left(j \phi-(j+2) \phi^{\prime}+\bar{\phi}_{j,+2}^{(1)}\right)+\sum_{j=1}^{6} \alpha_{j, 0}^{(1)} \cos \left(j \phi-j \phi^{\prime}+\bar{\phi}_{j, 0}^{(1)}\right) \\
& \left.\left.+\sum_{j=1}^{10} \alpha_{j,-4}^{(1)} \cos \left(j \phi-(j-4) \phi^{\prime}+\bar{\phi}_{j,-4}^{(1)}\right)+\sum_{j=1}^{2} \alpha_{j,+4}^{(1)} \cos \left(j \phi-(j+4) \phi^{\prime}+\bar{\phi}_{j,+4}^{(1)}\right)\right]\right\}
\end{aligned}
$$

A cursory look at the above equation may give the impression that the summation indices in various sums are terminated in an arbitrary manner. Interestingly, we find a possible way to predict the maximum value that $\mathrm{j}$ index can take in each of the above summations. This is related to the argument of $\phi^{\prime}$ in each of these cosine series. We infer that the argument of $\phi^{\prime}$ can take a maximum value of six as we are restricting eccentricity contributions to sixth order in $e_{t}$. This ensures that $j$ index can take maximum values of 8,6 and 4 at the Newtonian order in the above expression. In other words, $j_{\max }$ in the above expression is given such that $j_{\max } \pm n=6$ where $\pm n$ value arises from the the argument of $\phi^{\prime}$ variable in various summations. It is easy to see that the above relation holds true even at 0.5 and $1 \mathrm{PN}$ orders and it provides a natural check on the structure of these higher order PN contributions to $h(t)$.

To obtain GW response function for eccentric inspirals, we need to incorporate temporal evolution in $\omega, e_{t}, \phi$ and $\phi^{\prime}$, given by our earlier listed $3 \mathrm{PN}$-accurate differential equations. The fact that we require to solve the above four coupled differential equations numerically ensures that our approach to obtain ready-to-use $h(t)$ will be computationally expensive. This is clearly one 
of the motivation to obtain fully analytic $\tilde{h}(f)$ for compact binaries inspiraling along moderately eccentric orbits. Fortunately, we are in a position to compute analytic amplitude corrected $\tilde{h}(f)$ that incorporates $3 \mathrm{PN}$-accurate Fourier phase while keeping eccentricity contributions accurate to sixth order in $e_{0}$ at every PN order.

\section{ANALYTIC $\tilde{h}(f)$ FOR ECCENTRIC INSPIRALS WITH 1PN AMPLITUDE CORRECTIONS}

We first provide a detailed description of our approach to compute analytic Fourier transform of the restricted time domain inspiral family, given by Eq. (2.22). This will be followed by computing $\tilde{h}(f)$ associated with Eq. 2.32. Preliminary data analysis implications of our analytic $h(f)$ are probed in Sec. III B

\section{A. Approach to compute Fourier transform of $h(t)$ for compact binaries inspiraling along precessing eccentric orbits}

We begin by listing the expanded version of our quadrupolar order $h(t)$, namely Eq. 2.22 with $\mathcal{O}\left(e_{t}^{4}\right)$ eccentricity contributions as

$$
\begin{aligned}
h(t)= & \frac{G m \eta}{c^{2} D_{L}} x\left\{\left[\alpha_{1,-2}^{(0)} \cos \left(\phi+\phi^{\prime}+\bar{\phi}_{1,-2}^{(0)}\right)+\alpha_{2,-2}^{(0)} \cos \left(2 \phi+\bar{\phi}_{2,-2}^{(0)}\right)+\alpha_{3,-2}^{(0)} \cos \left(3 \phi-\phi^{\prime}+\bar{\phi}_{3,-2}^{(0)}\right)\right.\right. \\
& \left.+\alpha_{4,-2}^{(0)} \cos \left(4 \phi-2 \phi^{\prime}+\bar{\phi}_{4,-2}^{(0)}\right)+\alpha_{5,-2}^{(0)} \cos \left(5 \phi-3 \phi^{\prime}+\bar{\phi}_{5,-2}^{(0)}\right)+\alpha_{6,-2}^{(0)} \cos \left(6 \phi-4 \phi^{\prime}+\bar{\phi}_{6,-2}^{(0)}\right)\right] \\
& +\left[\alpha_{1,0}^{(0)} \cos \left(\phi-\phi^{\prime}+\bar{\phi}_{1,0}^{(0)}\right)+\alpha_{2,0}^{(0)} \cos \left(2 \phi-2 \phi^{\prime}+\bar{\phi}_{2,0}^{(0)}\right)+\alpha_{3,0}^{(0)} \cos \left(3 \phi-3 \phi^{\prime}+\bar{\phi}_{3,0}^{(0)}\right)+\alpha_{4,0}^{(0)} \cos \left(4 \phi-4 \phi^{\prime}+\bar{\phi}_{4,0}^{(0)}\right)\right] \\
& \left.+\left[\alpha_{1,+2}^{(0)} \cos \left(\phi-3 \phi^{\prime}+\bar{\phi}_{1,+2}^{(0)}\right)+\alpha_{2,+2}^{(0)} \cos \left(2 \phi-4 \phi^{\prime}+\bar{\phi}_{2,+2}^{(0)}\right)\right]\right\} .
\end{aligned}
$$

Clearly, we see three distinct square brackets that contain three cosine functions with explicitly time dependent arguments, namely $j \phi-(j-2) \phi^{\prime}, j \phi-j \phi^{\prime}$ and $j \phi-(j+2) \phi^{\prime}$. Note that $\alpha_{j, \pm n}^{(0)}$ and $\bar{\phi}_{j, \pm n}^{(0)}$ experience implicit temporal evolution due to the GW emission induced variations to $\omega$ and $e_{t}$. The main reason for displaying the above equation is to show explicitly how the periastron advance, defined by $\phi^{\prime}$, influences the harmonic structure of $h(t)$ in comparison with Eq. (4.21) of Ref. [44 or our Eq. (2.3).

We obtain an analytic Fourier domain version of the above equation with the help of the Stationary Phase Approximation, detailed in 62. How this approach can be employed to compute $\tilde{h}(f)$ for compact binaries spiraling along Keplerian eccentric orbits can be found in Sec. IV of Ref. 44. This approximation is quite appropriate for us as it provides a prescription to compute the asymptotic behavior of generalized cosine time series, as given by our Eq. (3.1). Without loss of any generality, we may write such a time series as

$$
S(t)=s(t) \cos (l \phi(t)),
$$

where $l>0$ and as expected $S(t)$ should be a product of slowly varying amplitude $s(t)$ and a rapidly varying cosine function with argument $l \phi(t)$. Due to the virtue of Riemann-Lebesgue lemma, as noted in Ref. 62, the Fourier transform of $S(t)$ becomes

$$
S_{f}(f)=\frac{1}{2} \int_{-\infty}^{\infty} s(t) e^{i f(2 \pi t-l \phi(t) / f)} d t .
$$

It is not difficult to gather that the argument of the exponential function vanishes at the stationary point $t_{0}$ such that $l \dot{\phi}\left(t_{0}\right)=2 \pi f$. This allows us to invoke the approach of SPA to obtain the asymptotic behaviour of $S_{f}(f)$ by the following expression:

$$
\begin{aligned}
S_{f}(f) & =s\left(t_{0}\right) e^{-i \Psi\left(t_{0}\right) \pm i \pi /(2 \times 2)}\left[\frac{2 !}{f\left|\Psi^{(2)}\left(t_{0}\right)\right|}\right]^{\frac{1}{2}} \frac{\Gamma(1 / 2)}{2} \\
& =\frac{s\left(t_{0}\right)}{2 \sqrt{l \dot{F}\left(t_{0}\right)}} e^{-i\left(\Psi\left(t_{0}\right) \mp \pi / 4\right)}
\end{aligned}
$$

where the Fourier phase is defined as

$$
\Psi(t):=-2 \pi f t+l \phi(t)
$$

Note that $F(t)=\dot{\phi}(t) / 2 \pi$ and therefore its value at the stationary point should be $F\left(t_{0}\right)=f / l$. Interestingly, a 
rather identical computation can be done to obtain the Fourier transform of a similar sinusoidal time series to be $i S_{f}(f)$.

To make operational the above expression for $S_{f}(f)$, we require an explicit expression for the above defined Fourier phase at the stationary point $t_{0}$, namely

$$
\Psi\left(t_{0}\right):=-2 \pi f t_{0}+l \phi\left(t_{0}\right)
$$

This is done by defining $\tau=F / \dot{F}$ such that $\phi(F)$ and $t(F)$ become

$$
\begin{aligned}
& \phi(F)=\phi_{c}+2 \pi \int^{F} \tau^{\prime} d F^{\prime}, \\
& t(F)=t_{c}+\int^{F} \frac{\tau^{\prime}}{F^{\prime}} d F^{\prime},
\end{aligned}
$$

where $\phi_{c}$ and $t_{c}$ are the orbital phase and time at coalescence. In the present context, $\tau$ is defined using our $3 \mathrm{PN}$-accurate expression for $\dot{\omega}$ given by Eq. 2.25). Additionally, we require $3 \mathrm{PN}$-accurate $e_{t}\left(\omega, \omega_{0}, e_{0}\right)$ expression, namely 3PN extension of Eq. (2.16), for computing these integrals analytically. The expression for $\Psi\left[F\left(t_{0}\right)\right]$ obtained using Eq. (3.6) and (3.7) in (3.5) may be written as

$$
\Psi_{l}\left[F\left(t_{0}\right)\right]=l \phi_{c}-2 \pi f t_{c}+2 \pi \int^{F\left(t_{0}\right)} \tau^{\prime}\left(l-\frac{f}{F^{\prime}}\right) d F^{\prime},
$$

where $F\left(t_{0}\right)=f / l$. In the present context, we need to evaluate the above integral at a point of time where the orbital frequency is related to the Fourier frequency by
$F\left(t_{0}\right)=f / l$. A close inspection of Eq. 3.1 reveals that our expression for the quadrupolar order time domain response function is structurally similar to the above displayed cosine time series and therefore we can easily adapt these results to obtain the Fourier transform of our quadrupolar order $h(t)$. However, the SPA based $\tilde{h}(f)$ will have contributions from a number of distinct stationary points. This is primarily due to the fact that Eq. (3.1) consists of cosine functions of three different arguments, namely $j \phi-(j+2) \phi^{\prime}, j \phi-(j-2) \phi^{\prime}$ and $j \phi-j \phi^{\prime}$. Note that there are only three distinct types of cosine arguments as we restricted our attention to the quadrupolar order GW response function for eccentric inspirals. However, we infer from our $1 \mathrm{PN}$-accurate $h(t)$, given by Eq. 2.32), that there are nine distinct types of cosine functions with arguments $j \phi-(j \pm n) \phi^{\prime}$ where $n=0,1,2,3,4$. The associated nine stationary points $t^{ \pm n}$ are computed by demanding that $\dot{\Psi}^{ \pm n}\left(t^{ \pm n}\right)=0$, where $\Psi^{ \pm n}(t):=-2 \pi f t+j \phi-(j \pm n) \phi^{\prime}$.

For computing Fourier transform of Eq.(3.1), we solve $\dot{\Psi}^{ \pm n}\left(t^{ \pm n}\right)=0$ to get the relevant stationary points and this leads to

$$
-2 \pi f+j \dot{\phi}-(j \pm n) \dot{\phi}^{\prime}=0,
$$

where $\dot{\phi}=N(1+k)$ and this by definition is $\omega$. The treatment of $\dot{\phi}^{\prime}$ requires PN approximation as $\dot{\phi}^{\prime}$ equals $k N$ ( this is because $\left.\phi^{\prime}=k l\right)$. We need to express $k N$ in terms of $\omega$ and this leads to $\dot{\phi}^{\prime}=\omega k /(1+k)$ as $\omega=N(1+k)$. For computing Fourier phase analytically, we express $\dot{\phi}^{\prime}$ as $\omega k_{(3)}^{(6)}$, where $k_{(3)}^{(6)}$ stands for the $3 \mathrm{PN}$-accurate expression for $k /(1+k)$ that incorporates $e_{t}$ contributions accurate to $\mathcal{O}\left(e_{t}^{6}\right)$. The resulting expression reads

$$
\begin{aligned}
k_{(3)}^{(6)}= & x\left\{3\left[1+e_{t}^{2}+e_{t}^{4}+e_{t}^{6}\right]\right\}+x^{2}\left\{\frac{9}{2}-7 \eta+\left[\frac{87}{4}-\frac{41 \eta}{2}\right] e_{t}^{2}+[39-34 \eta] e_{t}^{4}+\left[\frac{225}{4}-\frac{95 \eta}{2}\right] e_{t}^{6}\right\}+x^{3}\left\{\frac{27}{2}\right. \\
& +\left(-\frac{481}{4}+\frac{123 \pi^{2}}{32}\right) \eta+7 \eta^{2}+\left[\frac{519}{4}+\left(-\frac{2037}{4}+\frac{1599 \pi^{2}}{128}\right) \eta+61 \eta^{2}\right] e_{t}^{2}+\left[\frac{2811}{8}+\left(-1174+\frac{3321 \pi^{2}}{128}\right) \eta\right. \\
& \left.\left.+\frac{1361}{8} \eta^{2}\right] e_{t}^{4}+\left[\frac{10779}{16}+\left(-\frac{16901}{8}+\frac{2829 \pi^{2}}{64}\right) \eta+\frac{2675}{8} \eta^{2}\right] e_{t}^{6}\right\} .
\end{aligned}
$$

With the help of these inputs, the stationary points $t^{ \pm n}, \quad$ by

where $\dot{\Psi}^{ \pm n}\left(t^{ \pm n}\right)$ vanish, are given by

$$
\left(j-(j \pm n) k_{(3)}^{(6)}\right) \dot{\phi}\left(t^{ \pm n}\right)=2 \pi f .
$$

In other words, the stationary phase condition is given

$$
F\left(t^{ \pm n}\right)=\frac{f}{\left(j-(j \pm n) k_{(3)}^{(6)}\right)} .
$$

Rewriting $\Psi^{ \pm n}(t):=-2 \pi f t+j \phi-(j \pm n) \phi^{\prime}$ using relation between $\phi^{\prime}$ and $\phi\left(\phi^{\prime}=k_{(3)}^{(6)} \phi\right)$ gives $\Psi^{ \pm n}(t):=$ $-2 \pi f t+\left(j-(j \pm n) k_{(3)}^{(6)}\right) \phi$. We are now in a position to obtain analytic PN-accurate expressions for Fourier phases, associated with these stationary points. With Eq. (3.6) and (3.7), our Eq. (3.8) becomes 


$$
\Psi_{j}^{ \pm n}\left[F\left(t^{ \pm n}\right)\right]=\left(j-(j \pm n) k_{(3)}^{(6)}\right) \phi_{c}-2 \pi f t_{c}+2 \pi \int^{F\left(t^{ \pm n}\right)} \tau^{\prime}\left(j-(j \pm n) k_{(3)}^{(6)}-\frac{f}{F^{\prime}}\right) d F^{\prime}
$$

Note that $n$ takes values 0 and 2 as we are dealing with quadrupolar order GW response function given by Eq. (3.1). However, $n$ varies from 0 to 4 if the underlying GW response function contains $1 \mathrm{PN}$-accurate amplitude corrections that include at each PN order eccentricity corrections accurate to $\mathcal{O}\left(e_{t}^{6}\right)$. Further, we do not display here $3 \mathrm{PN}$-accurate expression for $\tau$ that includes the leading order $e_{t}$ corrections, listed as Eqs. (6.7a) and (6.7b) in Ref. 63. However, we do list below the explicit $3 \mathrm{PN}$-accurate $\Psi_{j}^{ \pm n}\left[F\left(t^{ \pm n}\right)\right]$ that incorporates leading order $e_{0}$ contributions at each $\mathrm{PN}$ order:

$$
\begin{aligned}
& \Psi_{j}^{n}=\left(j-(j+n) k_{(3)}^{(6)}\right) \phi_{c}-2 \pi f t_{c}-\frac{3 j}{256 \eta x^{5 / 2}}\left\{1-\frac{2355}{1462} e_{0}^{2} \chi^{-19 / 9}+x\left[-\frac{2585}{756}-\frac{25 n}{3 j}+\frac{55}{9} \eta\right.\right. \\
& \left.\left.+\left(\left(\frac{69114725}{14968128}+\frac{1805 n}{172 j}-\frac{128365}{12432} \eta\right) \chi^{-19 / 9}+\left(-\frac{2223905}{491232}+\frac{15464}{17544} \eta\right) \chi^{-25 / 9}\right) e_{0}^{2}\right]\right\}+x^{3 / 2}[-16 \pi \\
& \left.+\left(\frac{65561 \pi}{4080} \chi^{-19 / 9}-\frac{295945 \pi}{35088} \chi^{-28 / 9}\right) e_{0}^{2}\right]+x^{2}\left[-\frac{48825515}{508032}-\frac{31805 n}{252 j}+\left(\frac{22105}{504}-\frac{10 n}{j}\right) \eta+\frac{3085}{72} \eta^{2}\right. \\
& +\left(\left(\frac{115250777195}{2045440512}+\frac{323580365 n}{5040288 j}+\left(-\frac{72324815665}{6562454976}+\frac{36539875 n}{1260072 j}\right) \eta-\frac{10688155}{294624} \eta^{2}\right) \chi^{-19 / 9}\right. \\
& +\left(\frac{195802015925}{15087873024}+\frac{5113565 n}{173376 j}+\left(-\frac{3656612095}{67356576}-\frac{355585 n}{6192 j}\right) \eta+\frac{25287905}{447552} \eta^{2}\right) \chi^{-25 / 9}+\left(\frac{936702035}{1485485568}\right. \\
& \left.\left.\left.+\frac{3062285}{260064} \eta-\frac{14251675}{631584} \eta^{2}\right) \chi^{-31 / 9}\right) e_{0}^{2}\right]+x^{5 / 2}\left[\frac{14453 \pi}{756}-\frac{32 \pi n}{j}-\frac{65 \pi}{9} \eta-\left(\frac{1675}{756}+\frac{160 n}{3 j}+\frac{65}{9} \eta\right) \pi \log \left(\frac{f}{j}\right)\right. \\
& +\left(\left(-\frac{458370775 \pi}{6837264}-\frac{4909969 \pi n}{46512 j}+\frac{15803101 \pi \eta}{229824}\right) \chi^{-19 / 9}+\left(\frac{185734313 \pi}{4112640}-\frac{12915517 \pi \eta}{146880}\right) \chi^{-25 / 9}\right. \\
& \left.\left.+\left(\frac{26056251325 \pi}{1077705216}+\frac{680485 \pi n}{12384 j}-\frac{48393605 \pi \eta}{895104}\right) \chi^{-28 / 9}+\left(-\frac{7063901 \pi}{520128}+\frac{149064749 \pi \eta}{2210544}\right) \chi^{-34 / 9}\right) e_{0}^{2}\right] \\
& +x^{3}\left[\frac{13966988843531}{4694215680}+\frac{257982425 n}{508032 j}-\frac{640 \pi^{2}}{3}-\frac{6848 \gamma}{21}+\left(-\frac{20562265315}{3048192}-\frac{2393105 n}{1512 j}+\frac{23575 \pi^{2}}{96}\right.\right. \\
& \left.+\frac{1845 \pi^{2} n}{32 j}\right) \eta+\left(\frac{110255}{1728}+\frac{475 n}{24 j}\right) \eta^{2}-\frac{127825 \eta^{3}}{1296}-\frac{13696 \log (2)}{21}-\frac{3424 \log (x)}{21} \\
& +\left(\left(\frac{4175723876720788380517}{5556561877278720000}+\frac{534109712725265 n}{2405438042112 j}-\frac{21508213 \pi^{2}}{276480}-\frac{734341 \gamma}{16800}+\left(-\frac{37399145056383727}{28865256505344}\right.\right.\right. \\
& \left.-\frac{1219797059185 n}{2045440512 j}+\frac{12111605 \pi^{2}}{264192}+\frac{639805 n \pi^{2}}{22016 j}\right) \eta+\left(-\frac{159596464273381}{1718170030080}+\frac{43766986495 n}{1022720256 j}\right) \eta^{2}-\frac{69237581}{746496} \eta^{3} \\
& \left.-\frac{9663919 \log (2)}{50400}+\frac{4602177 \log (3)}{44800}-\frac{734341 \log (x)}{33600}\right) \chi^{-19 / 9}+\left(\frac{326505451793435}{2061804036096}+\frac{916703174045 n}{5080610304 j}\right. \\
& \left.-\left(\frac{13467050491570355}{39689727694848}+\frac{9519440485 n}{35282016 j}\right) \eta-\left(\frac{2186530635995}{52499639808}+\frac{7198355375 n}{45362592 j}\right) \eta^{2}+\frac{2105566535}{10606464} \eta^{3}\right) \chi^{-25 / 9} \\
& +\frac{24716497 \pi^{2}}{293760} \chi^{-28 / 9}+\left(-\frac{82471214720975}{45625728024576}-\frac{2153818055 n}{524289024 j}+\left(-\frac{48415393035455}{1629490286592}-\frac{119702185 n}{1560384 j}\right) \eta\right. \\
& \left.+\left(\frac{906325428545}{6466231296}+\frac{32769775 n}{222912 j}\right) \eta^{2}-\frac{2330466575}{16111872} \eta^{3}\right) \chi^{-31 / 9}+\left(-\frac{4165508390854487}{16471063977984}-\frac{96423905 \pi^{2}}{5052672}\right. \\
& +\frac{2603845 \gamma}{61404}+\left(-\frac{1437364085977}{53477480448}+\frac{3121945 \pi^{2}}{561408}\right) \eta+\frac{4499991305 \eta^{2}}{636636672}+\frac{2425890995 \eta^{3}}{68211072}+\frac{1898287 \log (2)}{184212}
\end{aligned}
$$




$$
\left.\left.\left.+\frac{12246471 \log (3)}{163744}+\frac{2603845 \log (x)}{122808}-\frac{2603845 \log (\chi)}{184212}\right) \chi^{-37 / 9}\right) e_{0}^{2}\right] .
$$

A few comments are in order. To obtain the circular limit, we require to impose $n=j$ in $j \phi-(j-n) \phi^{\prime}$ and let $e_{0}=0$. This is indeed due to the fact that $k_{(3)}^{(6)}$ does not go to zero in the circular limit. Additionally, we have verified that the resulting $\Psi_{2}^{-2}(f)$ expression in the $e_{0} \rightarrow 0$ limit is identical to $3 \mathrm{PN}$ accurate version of Eq. (6.26) in Ref. 63. while neglecting the spin contributions. It is natural to expect that the $\Psi_{j}^{0}(f)$ version of our above equation should be identical to Eq. (6.26) of Ref. 63. This is because this equation indeed provided quadrupolar $\tilde{h}(f)$ with $3 \mathrm{PN}$-accurate Fourier phase while incorporating leading order $e_{0}$ corrections at each $\mathrm{PN}$ order by extending the post-circular approach of Ref. 44 . However, our expression for $\Psi_{j}^{0}(f)$ is not identical to Eq. (6.26) of Ref. 63. This is because that effort did not incorporate the effect of periastron advance while obtaining analytic expression for their Fourier phase. A close inspection of the $n=0$ version of our Eq. (3.12) reveals that it will still be influenced by our PN-accurate expres- sion for $k_{(3)}^{(6)}$. This clearly shows that it is rather impossible to remove the effect of periastron advance from our Eq. (3.12). Therefore, our Eq. (3.13) will be different from Eq. (6.26) of Ref. 63 which, as noted earlier, neglected the effect of periastron advance. The differences may be attributed to the physical fact that we are providing an analytic expression for $\tilde{h}(f)$ associated with compact binaries inspiraling along PN-accurate eccentric orbits. In contrast, Ref. 63 models inspiral GWs from compact binaries spiraling in along Newtonian orbits though frequency evolution in both cases are fully $3 \mathrm{PN}$ accurate. Additionally, we are unable to match with the $2 \mathrm{PN}$ order results of Ref. [49] due to similar reasons. We note in passing that the explicit $3 \mathrm{PN}$-accurate $\mathcal{O}\left(e_{0}^{4}\right)$ contributions to $\Psi_{j}^{n}(f)$ and the associated $3 \mathrm{PN}$ accurate $e_{t}$ expression are provided in the Appendix C.

We now employ fully the final result of SPA, namely Eq. (3.4), to compute the Fourier transform of Eq. 3.1. This gives us

$$
\begin{aligned}
\tilde{h}\left[F\left(t_{0}\right)\right]= & \left(\frac{5 \pi \eta}{384}\right)^{1 / 2} \frac{G^{2} m^{2}}{c^{5} D_{L}}\left(\frac{G m \pi 2 F\left(t_{0}\right)}{c^{3}}\right)^{-7 / 6} \frac{\left(1-e_{t}^{2}\right)^{7 / 4}}{\left(1+\frac{73}{24} e_{t}^{2}+\frac{37}{96} e_{t}^{4}\right)^{1 / 2}}\left\{\sum_{j=1}^{6} \alpha_{j,-2}^{(0)} \sqrt{\frac{2}{j}} e^{-i \bar{\phi}_{j,-2}^{(0)}\left[F\left(t_{0}\right)\right]} e^{-i\left(\Psi_{j}^{-2}+\pi / 4\right)}\right. \\
& \left.+\sum_{j=1}^{4} \alpha_{j, 0}^{(0)} \sqrt{\frac{2}{j}} e^{-i \bar{\phi}_{j, 0}^{(0)}\left[F\left(t_{0}\right)\right]} e^{-i\left(\Psi_{j}^{0}+\pi / 4\right)}+\sum_{j=1}^{2} \alpha_{j,+2}^{(0)} \sqrt{\frac{2}{j}} e^{-i \bar{\phi}_{j,+2}^{(0)}\left[F\left(t_{0}\right)\right]} e^{-i\left(\Psi_{j}^{+2}+\pi / 4\right)}\right\}
\end{aligned}
$$

where we have used the quadrupolar (Newtonian) order differential equation for the orbital frequency, available in Refs. [34, 55], to compute the amplitudes of $\tilde{h}\left[F\left(t_{0}\right)\right]$. Note that we require to employ the earlier defined stationary points to replace $F\left(t_{0}\right)$. In practice, we employ the unperturbed stationary points, namely $F\left(t_{0}\right)=f / j$, while evaluating the amplitudes of $\tilde{h}(f)$.

In what follows, we collect the above pieces together to display the quadrupolar order $\tilde{h}(f)$ that incorporates fourth order orbital eccentricity contributions while including the effects due to $3 \mathrm{PN}$-accurate frequency, eccentricity evolution and periastron advance as

$$
\begin{aligned}
\tilde{h}(f) & =\left(\frac{5 \pi \eta}{384}\right)^{1 / 2} \frac{G^{2} m^{2}}{c^{5} D_{L}}\left(\frac{G m \pi f}{c^{3}}\right)^{-7 / 6}\left\{\sum_{j=1}^{6} \xi_{j,-2}^{(0)}\left(\frac{j}{2}\right)^{2 / 3} e^{-i\left(\Psi_{j}^{-2}+\frac{\pi}{4}\right)}+\sum_{j=1}^{4} \xi_{j, 0}^{(0)}\left(\frac{j}{2}\right)^{2 / 3} e^{-i\left(\Psi_{j}^{0}+\frac{\pi}{4}\right)}\right. \\
& \left.+\sum_{j=1}^{2} \xi_{j,+2}^{(0)}\left(\frac{j}{2}\right)^{2 / 3} e^{-i\left(\Psi_{j}^{+2}+\frac{\pi}{4}\right)}\right\}
\end{aligned}
$$

where the Fourier amplitudes $\xi_{j, \pm n}^{(0)}$ are now given by

$$
\xi_{j, \pm n}^{(0)}=\frac{\left(1-e_{t}^{2}\right)^{7 / 4}}{\left(1+\frac{73}{24} e_{t}^{2}+\frac{37}{96} e_{t}^{4}\right)^{1 / 2}} \alpha_{j, \pm n}^{(0)} e^{-i \bar{\phi}_{j, \pm n}^{(0)}(f / j)},
$$

and $n$ takes values 0 and 2. A crucial expression that will be required to operationalize the above $\tilde{h}(f)$, namely $3 \mathrm{PN}$-accurate expression for $e_{t}$ in terms of $e_{0}, x$ and $\chi$, 
is listed as Eq. C1 in the Appendix. Note that the approach to obtain such an expression for $e_{t}$ is detailed in Ref. 49] and briefly summarized in Sec. II A. Finally, the fully $3 \mathrm{PN}$-accurate expression for $\Psi_{j}^{n}(f)$ that incorporates fourth order orbital eccentricity contributions at each PN order is displayed as Eq. (C5) in the Appendix. It should be noted that the approach of SPA demands the evaluation of Fourier amplitudes, $\xi_{j, \pm n}$ and Fourier phases, $\Psi_{j}^{ \pm n}$ at $F\left(t^{ \pm n}\right)=f /\left(j-(j \pm n) k_{(3)}^{(6)}\right)$

We have extended these calculations by including $1 \mathrm{PN}$ accurate amplitude corrections to $h_{\times}$and $h_{+}$with the help of Eqs. 2.27, 2.28a, 2.28b and 2.28c. Additionally, we have included initial eccentricity corrections, accurate to $\mathcal{O}\left(e_{0}^{6}\right)$, in our $3 \mathrm{PN}$-accurate $e_{t}$ and $\Psi_{j}^{n}(f)$ expressions. We note in passing that these expressions are available in the accompanying Mathematica file. The resulting expression for $\tilde{h}(f)$ may be symbolically written as

$$
\begin{aligned}
\tilde{h}(f)= & \left(\frac{5 \pi \eta}{384}\right)^{1 / 2} \frac{G^{2} m^{2}}{c^{5} D_{L}}\left(\frac{G m \pi f}{c^{3}}\right)^{-7 / 6}\left\{\left[\sum_{j=1}^{6} \xi_{j, 0}^{(0)}\left(\frac{j}{2}\right)^{2 / 3} e^{-i\left(\Psi_{j}^{0}+\frac{\pi}{4}\right)}+\sum_{j=1}^{4} \xi_{j,+2}^{(0)}\left(\frac{j}{2}\right)^{2 / 3} e^{-i\left(\Psi_{j}^{+2}+\frac{\pi}{4}\right)}\right.\right. \\
& \left.+\sum_{j=1}^{8} \xi_{j,-2}^{(0)}\left(\frac{j}{2}\right)^{2 / 3} e^{-i\left(\Psi_{j}^{-2}+\frac{\pi}{4}\right)}\right]+\left(\frac{G m \pi f}{c^{3}}\right)^{1 / 3} \delta\left[\sum_{j=1}^{5} \xi_{j,+1}^{(0.5)}\left(\frac{j}{2}\right)^{1 / 3} e^{-i\left(\Psi_{j}^{+1}+\frac{\pi}{4}\right)}\right. \\
& \left.+\sum_{j=1}^{7} \xi_{j,-1}^{(0.5)}\left(\frac{j}{2}\right)^{1 / 3} e^{-i\left(\Psi_{j}^{-1}+\frac{\pi}{4}\right)}+\sum_{j=1}^{3} \xi_{j,+3}^{(0.5)}\left(\frac{j}{2}\right)^{1 / 3} e^{-i\left(\Psi_{j}^{+3}+\frac{\pi}{4}\right)}+\sum_{j=1}^{9} \xi_{j,-3}^{(0.5)}\left(\frac{j}{2}\right)^{1 / 3} e^{-i\left(\Psi_{j}^{-3}+\frac{\pi}{4}\right)}\right] \\
& +\left(\frac{G m \pi f}{c^{3}}\right)^{2 / 3}\left[\sum_{j=1}^{6} \xi_{j, 0}^{(1)} e^{-i\left(\Psi_{j}^{0}+\frac{\pi}{4}\right)}+\sum_{j=1}^{4} \xi_{j,+2}^{(1)} e^{-i\left(\Psi_{j}^{+2}+\frac{\pi}{4}\right)}+\sum_{j=1}^{8} \xi_{j,-2}^{(1)} e^{-i\left(\Psi_{j}^{-2}+\frac{\pi}{4}\right)}\right. \\
& \left.\left.+\sum_{j=1}^{2} \xi_{j,+4}^{(1)} e^{-i\left(\Psi_{j}^{+4}+\frac{\pi}{4}\right)}+\sum_{j=1}^{10} \xi_{j,-4}^{(1)} e^{-i\left(\Psi_{j}^{-4}+\frac{\pi}{4}\right)}\right]\right\} .
\end{aligned}
$$

In the above expression, the Fourier amplitudes are given by

$$
\begin{aligned}
\xi_{j, \pm n}^{(p)}=\{ & \frac{\left(1-e_{t}^{2}\right)^{7 / 4}}{\left(1+\frac{73}{24} e_{t}^{2}+\frac{37}{96} e_{t}^{4}\right)^{1 / 2}}+\frac{\left(1-e_{t}^{2}\right)^{3 / 4}}{10752\left(1+\frac{73}{24} e_{t}^{2}+\frac{37}{96} e_{t}^{4}\right)^{3 / 2}}\left[11888+14784 \eta-e_{t}^{2}(87720-159600 \eta)\right. \\
& \left.\left.-e_{t}^{4}(171038-141708 \eta)-e_{t}^{6}(11717-8288 \eta)\right]\right\} \alpha_{j, \pm n}^{(p)} e^{-i \bar{\phi}_{j, \pm n}^{(p)}}
\end{aligned}
$$

where the superscript $p$ takes values $0,0.5$ and 1 in our amplitude corrected $\tilde{h}(f)$. Further, we have used the $1 \mathrm{PN}$-accurate differential equation for the orbital fre- quency while obtaining the Fourier amplitude expressions. This expression, adaptable from Eqs. (B8a) and (B9a) of Ref. 49, reads

$$
\begin{aligned}
\frac{d F}{d t}= & \frac{48 c^{6} \eta}{5 \pi G^{2} m^{2}}\left(\frac{G m 2 \pi F}{c^{3}}\right)^{11 / 3} \frac{\left(1+\frac{73}{24} e_{t}^{2}+\frac{37}{96} e_{t}^{4}\right)}{\left(1-e_{t}^{2}\right)^{7 / 2}}-\frac{743 c^{6} \eta}{35 \pi G^{2} m^{2}}\left(\frac{G m 2 \pi F}{c^{3}}\right)^{13 / 3} \frac{1}{\left(1-e_{t}^{2}\right)^{9 / 2}} \\
& \left\{1+\frac{924}{743} \eta+e_{t}^{2}\left(-\frac{10965}{1486}+\frac{9975}{743} \eta\right)+e_{t}^{4}\left(-\frac{85519}{5944}+\frac{35427}{2972} \eta\right)+e_{t}^{6}\left(-\frac{11717}{11888}+\frac{518}{743} \eta\right)\right\}
\end{aligned}
$$

The explicit expressions for $e_{t}$ and $\Psi_{j}^{n}(f)$ that incorpo- 
order, as noted earlier, are listed in the Appendix C

We move on to contrast our approach with other attempts in the literature. The Sec. VI of Ref. 44 indeed sketched a road map to include PN corrections to their Newtonian waveform family. This road map included a suggestion to incorporate the effect of periastron advance into their quadrupolar order GW polarization states, influenced by Ref. 34. Their suggestion involves splitting the orbital phase evolution into two parts where one part remains linear in the mean anomaly $l$ while the other part is periodic in $l$. These considerations influenced them to re-write our Eq. 2.3) essentially to be

$$
h(t)=-\frac{G m \eta}{c^{2} D_{L}} x \sum_{j=1}^{10} \alpha_{j} \cos \left\{j l\left(1+k_{(1)}^{(6)}\right)+\phi_{j}\right\},
$$

where $k_{(1)}^{(6)}$ stands for the $1 \mathrm{PN}$ accurate expression for $k$, given by $3 x /\left(1-e_{t}^{2}\right)$, expanded to the sixth order in $e_{t}$ (see our Eq.(3.10)). It is not difficult to see that the associated SPA based Fourier phase takes the following form:

$$
\Psi_{j}(F)=\lambda[t(f / j)]-2 \pi f t(f / j),
$$

where

$$
\begin{gathered}
\lambda[t(f / j)]=j \phi_{c}+j \int^{f / j} \frac{\dot{\lambda}^{\prime}}{\dot{F}^{\prime}} d F^{\prime} \\
t(f / j)=t_{c}+\int^{f / j} \frac{d F^{\prime}}{\dot{F}^{\prime}} .
\end{gathered}
$$

It turned out that $\dot{\lambda}^{\prime} \equiv \omega$ by construction. The use of $\omega$ in the above Fourier phase expression essentially ensures that the suggestion of Ref. [44] leads to what is detailed in Ref. [49. Note that Ref. [49] provided $\tilde{h}(f)$ in terms of infinite set of harmonics with quadrupolar order amplitudes and $2 \mathrm{PN}$-accurate Fourier phase. We observe that Ref. 44 indeed commented on the absence of side bands in their prescription in comparison with what was reported in Refs. [66, 67] and suggested future investigations to clarify the issue. In contrast, the present investigation employs Eqs. 2.32 that explicitly incorporates the effect of periastron advance both in the amplitude and phase of GW polarization states, as detailed in Ref. [45. The use of such an expression ensures that our analytic Fourier domain expression does indeed contain periastron advance induced frequency side bands. Additionally, Refs. 29, 59] employed the dominant order periastron advance induced decomposition of Fourier phases, associated with quadrupolar order gravitational waveform, while exploring LISA and aLIGO relevant parameter estimation studies. A close comparison of Eqs. (B10) and (B11) of Ref. [59] and Eqs. (35) and
(36) of Ref. 29] with our Eq. (3.10) reveals fairly identical expressions for the Fourier phases. These considerations allowed us to state that our expression for $\tilde{h}(f)$, given by Eqs. (3.17), (3.18), (C1), (C5), provides analytic $\mathrm{PN}$-accurate Fourier domain templates for compact binaries inspiraling along $\mathrm{PN}$-accurate precessing eccentric orbits. We are now in a position to explore basic GW data analysis implications of our inspiral templates.

\section{B. Preliminary GW data analysis implications}

We employ the familiar match computations to probe basic GW data analysis implications of our PN-accurate inspiral templates. Following Ref. [52, the match $\mathcal{M}\left(h_{s}, h_{t}\right)$ between members of two waveform classes, namely signal $h_{s}$ and template $h_{t}$, is computed by maximizing a certain overlap integral $\mathcal{O}\left(h_{s}, h_{t}\right)$ with respect to the kinematic variables of the template waveform. In other words,

$$
\mathcal{M}\left(h_{s}, h_{t}\right)=\max _{t_{0}, \phi_{0}} \mathcal{O}\left(h_{s}, h_{t}\right),
$$

where $t_{0}$ and $\phi_{0}$ are the detector arrival time and the associated arrival phase of our template. The overlap integral involves the interferometer-specific normalized inner product between members of $h_{s}$ and $h_{t}$ families; it reads

$$
\left\langle h_{s} \mid h_{t}\right\rangle=4 \operatorname{Re} \int_{f_{\text {low }}}^{f_{\text {high }}} \frac{\tilde{h}_{s}^{*}(f) \tilde{h}_{t}(f)}{S_{\mathrm{h}}(f)} d f,
$$

where $\tilde{h}_{s}(f)$ and $\tilde{h}_{t}(f)$ are the Fourier transforms of the $h_{s}(t)$ and $h_{t}(t)$ inspiral waveforms. Further, $S_{\mathrm{h}}(f)$ denotes the one-sided power spectral density of the detector noise. In the following, we employ the zero-detuned, high power (ZDHP) noise configuration of Advanced LIGO at design sensitivity 68. In our $\mathcal{M}$ estimates, we let $f_{\text {low }}$ be $20 \mathrm{~Hz}$, corresponding to the lower cut-off frequency of Advanced LIGO. The upper frequency limit $f_{\text {high }}$ is chosen to be the usual $f_{\mathrm{LSO}}=c^{3} /\left(G m \pi 6^{3 / 2}\right)$ of the last stable circular orbit. We have verified that orbital eccentricities of compact binaries reduce to well below $10^{-2}$ at $f_{\text {high }}=f_{\text {LSO }}$, thereby justifying the use of the last stable circular orbit frequency for the upper frequency limit.

We require additional steps to operationalize our inspiral templates while performing the $\mathcal{M}$ computations. Clearly, these waveform families should only be implemented within the physically allowed frequency intervals. This is to ensure that the many higher harmonics present in these waveform families do not cross the above listed upper frequency limit. Influenced by Ref. [44, we invoke the Unit Step function $(\Theta)$ to operationalize our inspiral templates. This step function allows us to appropriately terminate the waveform as $\Theta(y)=1$ for $y \geq 0$ and zero otherwise. The structure of our quadrupolar amplitude inspiral family, given by Eqs. 3.15), compels us to invoke $\Theta$ functions such that 


$$
\begin{aligned}
\tilde{h}(f)= & \left(\frac{5 \pi \eta}{384}\right)^{1 / 2} \frac{G^{2} m^{2}}{c^{5} D_{L}}\left(\frac{G m \pi f}{c^{3}}\right)^{-7 / 6}\left\{\sum_{j=1}^{4} \xi_{j, 0}^{(0)}\left(\frac{j}{2}\right)^{2 / 3} e^{-i\left(\frac{\pi}{4}+\Psi_{j}^{0}\right)} \times \Theta\left[\left(j-j k_{(3)}^{(6)}\right) f_{L S O}-2 f\right]\right. \\
& +\sum_{j=1}^{2} \xi_{j,+2}^{(0)}\left(\frac{j}{2}\right)^{2 / 3} e^{-i\left(\frac{\pi}{4}+\Psi_{j}^{+2}\right)} \times \Theta\left[\left(j-(j+2) k_{(3)}^{(6)}\right) f_{L S O}-2 f\right]+\sum_{j=1}^{6} \xi_{j,-2}^{(0)}\left(\frac{j}{2}\right)^{2 / 3} e^{-i\left(\frac{\pi}{4}+\Psi_{j}^{-2}\right)} \\
& \left.\times \Theta\left[\left(j-(j-2) k_{(3)}^{(6)}\right) f_{L S O}-2 f\right]\right\} .
\end{aligned}
$$

Note that we have appropriately shifted the upper frequency limits to ensure that higher harmonics are suitably terminated. While implementing our $\tilde{h}(f)$ we have encountered the violation of the stationary phase condition, namely Eq. (3.11), at a few Fourier frequencies corresponding to lower harmonic indices $(j \sim 1,2)$. We infer that the periastron advance induced shift of these harmonics can lead to negative GW frequencies. Therefore, we have discarded such Fourier components. Interestingly, Ref. [46] showed that these harmonics provide negligible contributions to the GW power spectrum, which may be used to justify our neglect of such Fourier components in the implementation of our waveform families. The above steps ensure smoothly varying templates which we will use in the following to pursue match computations. We provide three frequency series of the same length (corresponding to $h_{s}$ and $h_{t}$ inspiral families and the ZDHP noise power spectral density) and employ a routine from the free and open software package PyCBC [69] to compute various $\mathcal{M}$ estimates.

We qualify the implications of our match estimates on GW data analysis by considering the threshold $\mathcal{M}\left(h_{s}, h_{t}\right) \geq 0.97$, denoted in the presentation of results in Figs. 1, 2 and 3 by solid black lines. This limit corresponds to a loss of less than $10 \%$ of all signals in the matched filter searches. In regions of parameter space where the computed matches are high, i.e., $\mathcal{M} \geq 0.97$, waveform models are generally considered both effectual templates for the detection of fiducial GW signals and reasonably faithful in the estimation of GW source parameters [52]. However, even if $\mathcal{M}$ larger than 0.97 , certain errors in the model waveform (due to unmodeled effects of, e.g., eccentricity) may become distinguishable from noise at high signal-to-noise ratio (SNR) and can affect the accuracy of source parameter estimation. Negligible systematic errors in parameter estimation - despite differences between the true signal waveform and the template model - can be guaranteed only if $\left(h_{s}-h_{t}, h_{s}-h_{t}\right)<1$, the so-called indistinguishability criterion [70. In other words, such systematic errors in the estimated source parameters may become significant when the mismatch $1-\mathcal{M}_{c} \geq 1 / \mathrm{SNR}^{2}$ and clearly depend on the amplitude of the signal. In the following analysis, we let the signal-to-noise ratio of our fiducial GW signals be $\mathrm{SNR}=30$ (corresponding to the SNR of the binary neutron star inspiral GW170817) and probe the distinguishability of certain effects in our model waveforms for inspiraling eccentric binaries. In the inset plots of Figs. 1 and 2, we zoom into those regions of parameter space where we can expect waveform uncertainties to become indistinguishable from noise for $\mathrm{SNR}=30$; the corresponding distinguishable limit $\mathcal{M}_{c}$ is represented by the dashed black lines.

We first probe the importance of higher-order eccentricity corrections in the GW phasing. For this purpose, we let the signal family $h_{s}$ to be our quadrupolarorder $\tilde{h}(f)$, with a $3 \mathrm{PN}$-accurate Fourier phase that includes next-to-next-to leading order, $\mathcal{O}\left(e_{0}^{6}\right)$ eccentricity corrections at each PN order. The template family is given by a quadrupolar-order $\tilde{h}(f)$ in the low-eccentricity limit, incorporating only the leading-order, $\mathcal{O}\left(e_{0}^{2}\right)$ eccentricity contributions in the $3 \mathrm{PN}$-accurate Fourier phase. We consider the traditional non-spinning compact binary sources relevant for Advanced LIGO: namely, binary neutron stars (NS-NS), NS-BH systems and binary black holes $(\mathrm{BH}-\mathrm{BH})$, with NS and $\mathrm{BH}$ components of $1.4 M_{\odot}$ and $10 M_{\odot}$, respectively. For each of these three configurations, we compute the match between signal and template waveforms for different values of the initial orbital eccentricity $e_{0}$ between 0 and 0.4 (defined at the cut-off frequency $20 \mathrm{~Hz}$ ). Fig. 1 suggests that the importance of higher-order eccentricity corrections for GW data analysis is strongly dependent on the total mass of an eccentric compact binary source. Given the same $e_{0}$ but for configurations with increasing total mass, we find that templates restricted to leading-order eccentricity corrections become increasingly faithful representations of those inspiral waveforms that include higher-order eccentricity effects at each PN order. This is expected, as compact binaries with higher total mass provide a smaller number of inspiral GW cycles in the frequency window of Advanced LIGO. Therefore, these systems require larger initial eccentricities to bring on a substantial de-phasing and subsequent mismatch between our inspiral signal and template families. Fig. 1 indicates that a waveform model restricted to only leading-order eccentricity corrections would be an effectual template family for the detection of GWs from even moderately eccentric inspirals (with $e_{0} \leq 0.15$ and $\leq 0.3$ for our traditional NS-NS and BH-BH binaries, respectively). How- 
ever, the inset of Fig. 1 1 suggests that waveform effects of higher-order eccentricity corrections become distinguishable from detector noise at significantly lower initial eccentricities $\left(e_{0} \geq 0.07\right.$ and $\geq 0.17$ for GWs from NS$\mathrm{NS}$ and $\mathrm{BH}-\mathrm{BH}$ systems with $\mathrm{SNR}=30$ ). In this region of parameter space, we should expect systematic errors in source parameter estimation with inspiral templates that are accurate only to leading order in eccentricity $e_{0}$. The inclusion of higher-order eccentricity corrections in waveform modeling is therefore desirable for an accurate follow-up of eccentric GW signals.

We move on to probe data analysis implications of including the effect of periastron advance in our eccentric inspiral waveforms $\tilde{h}(f)$. In our match calculation $\mathcal{M}\left(h_{s}, h_{t}\right)$, the signal waveforms employ our quadrupolar-order $\tilde{h}(f)$ given by Eq. 3.15, including both $k$ and $e_{t}$ effects to the sixth order in $e_{0}$ at each PN order. We build a template family $h_{t}$ that neglects effects of periastron advance, by extending to $3 \mathrm{PN}$ order previously developed eccentric inspiral waveforms (provided with $2 \mathrm{PN}$-accurate Fourier phase in Ref. [49]). In other words, we construct quadrupolar templates $\tilde{h}_{t}(f)$ with the help of Eq. (2.5) and the 3PN extension of our Newtonian Eq. 2.11) for $\Psi_{j}$ while incorporating all $\mathcal{O}\left(e_{0}^{6}\right)$ corrections at each PN order. Additionally, we evaluate the Fourier phase at the unperturbed stationary point $F=f / j$ [44. It is important to note that such a template waveform family ignores the effect of periastron advance in its Fourier phase evolution. We consider the same NS-NS, NS-BH and BH-BH systems as before and compute the match between signal and template waveforms for discrete values of initial orbital eccentricity at $20 \mathrm{~Hz}, e_{0} \in[0,0.4]$. From our results, presented in Fig. 2, we learn that the significance of periastron advance effects for GW data analysis is rather independent of the total mass of the source, with similar match estimates for all three traditional compact binaries under consideration. Periastron advance starts to influence the effectualness of GW templates for detection only for systems that have eccentricities $e_{0}>0.25$ at $20 \mathrm{~Hz}$. This agrees with our observation that $k$-induced modulations in the inspiral waveforms presented in Fig. 5 and 6 of Ref. 34. become clearly visible only for moderate values of initial orbital eccentricity. However, we can expect systematic biases in the source parameter estimation for much smaller values of orbital eccentricity. The inset of Fig. 2 suggests that periastron advance effects in an eccentric GW signal with SNR $=30$ would already become distinguishable from noise for eccentricities $e_{0}>0.03$ at $20 \mathrm{~Hz}$, leading to systematic errors in the recovered source parameters when waveform models neglect periastron advance.

Lastly, we explore the relevance of PN-accurate amplitude corrections while constructing realistic analytic Fourier-domain waveforms for eccentric inspirals. For these $\mathcal{M}$ estimates, we invoke as the expected GW signal our $1 \mathrm{PN}$-accurate amplitude corrected $\tilde{h}(f)$, given by Eq. (3.17), including the effects of $3 \mathrm{PN}$-accurate perias- tron advance, frequency and eccentricity evolution accurate to sixth order in orbital eccentricity. For the template family, we are utilizing a quadrupolar-order $\tilde{h}(f)$, given by Eq. (3.15), that includes the same order effects of $3 \mathrm{PN}$-accurate periastron advance and $3 \mathrm{PN}$-accurate frequency and eccentricity evolution as above. We consider five compact binary configurations with a fixed total mass $m=m_{1}+m_{2}=20 M_{\odot}$ and varying mass ratios $q=m_{1} / m_{2} \in\{1,3,5,7,9\}$. For each of these configurations, we pursue match computations for different choices of initial orbital eccentricity $e_{0} \in[0,0.4]$ at $20 \mathrm{~Hz}$, resulting in Fig. 3. We observe that amplitude corrections are rather unimportant while constructing template waveforms for equal-mass binaries in eccentric orbits. This is expected, as the dominant amplitude corrections - appearing at $0.5 \mathrm{PN}$ order in Eq. 3.17) - are proportional to $\sqrt{1-4 \eta}$ and therefore vanish for equal-mass binaries. Our plots suggest that the effect of amplitude corrections on the faithfulness of eccentric inspiral waveforms crucially depends on the mass ratio of a binary system, with $\mathcal{M}$ rapidly dropping below the critical value of 0.97 as $q \geq 5$, even for systems with negligible initial eccentricities. This is a familiar result from the modeling of compact binary inspiral along circular orbits and points to the relevance of higher modes for GWs from binaries with asymmetric masses [71. In other words, our plots in Fig. 3 essentially confirm previous literature that compared restricted and amplitude-corrected $\tilde{h}(f)$ for quasi-circular inspiral. Interestingly, we find that the $q$-dependent effect of amplitude corrections on the faithfulness of eccentric inspiral waveforms is largely unaffected by the value of initial eccentricity $e_{0}$.

\section{CONCLUSIONS}

We have provided fully analytic PN-accurate Fourier domain gravitational waveforms for compact binaries inspiraling along precessing moderately eccentric orbits. Our inspiral approximant contains 1PN-accurate amplitude corrections and its Fourier phase incorporates the effects of 3PN-accurate periastron advance and GW emission. Additionally, the eccentricity effects are accurate to sixth order in $e_{0}$ at each PN order. We infer from our analytic waveform expression that the orbital eccentricity induced higher harmonics are no longer integer multiples of orbital frequency due to the influence of periastron advance. This substantiates and extends what is detailed in Ref. [45] for compact binaries inspiraling along PN-accurate precessing eccentric orbits. Preliminary GW data analysis implications of our waveforms are probed with the help of the usual match computations.

In what follows, we provide a step-by-step summary of our effort.

1. We start from our Eqs. 2.18 and 2.19) that provide quadrupolar order $\mathrm{GW}$ polarization states from compact binaries in $\mathrm{PN}$-accurate eccentric orbits as a sum over various harmonics. 


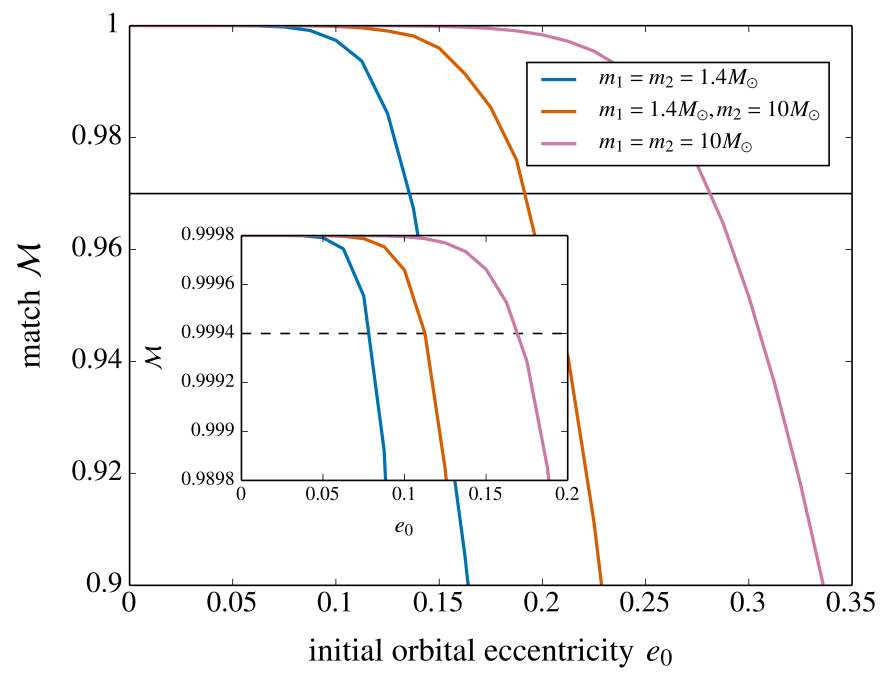

FIG. 1. Matches between eccentric waveform models with different orders of eccentricity corrections. We are comparing waveforms that take only leading-order $\mathcal{O}\left(e_{0}^{2}\right)$ eccentricity corrections into account to those that include eccentricity corrections up to next-to-next-to leading order $\mathcal{O}\left(e_{0}^{6}\right)$. We consider three configurations of a NS and a $\mathrm{BH}$ with masses of $1.4 M_{\odot}$ and $10 M_{\odot}$, respectively: i.e., NS-NS (blue curve), NS-BH (orange curve) and BH-BH (pink curve) systems. The initial orbital eccentricity $e_{0}$ refers to the eccentricity of the binary system at $20 \mathrm{~Hz}$. Given the same $e_{0}$, the effect of higher-order eccentricity corrections on the agreement between signal and template is strongly dependent on the total mass of the compact binary source. The solid black line denotes the threshold $\mathcal{M}=0.97$, associated with the effectualness of a model for GW detection and its faithfulness for source parameter estimation. The inset plot zooms into the region of parameter space where we can expect the effect of higher-order eccentricity corrections to become distinguishable from noise for SNR $=30$, leading to systematic errors in parameter estimation; the dashed black line represents the indistinguishability criterion.

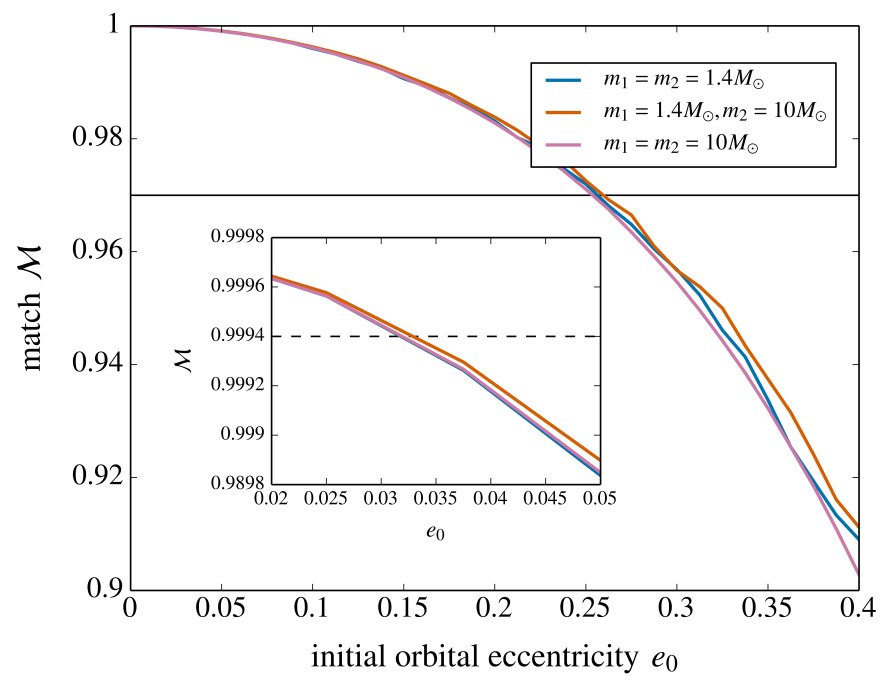

FIG. 2. Matches between eccentric waveform models that include or neglect effects of periastron advance. We consider the same three configurations of binaries with NS and BH components as in Fig. 11 i.e., NS-NS (blue curve), NS-BH (orange curve) and $\mathrm{BH}-\mathrm{BH}$ (pink curve) systems. The initial orbital eccentricity $e_{0}$ is again defined at the lower cut-off frequency $20 \mathrm{~Hz}$. We infer that the significance of periastron advance effects for GW data analysis is rather independent of the total mass of the source. We interpret our results by considering the threshold $\mathcal{M}=0.97$ (represented by the solid black line) below which a waveform model should be considered ineffectual for detection and unfaithful for parameter estimation. In the inset plots, we highlight the parameter space of small eccentricities to probe the importance of systematic errors in parameter estimation due to waveform uncertainties. The dashed black line represents the distinguishable limit for a fiducial GW signal with SNR $=30$. 


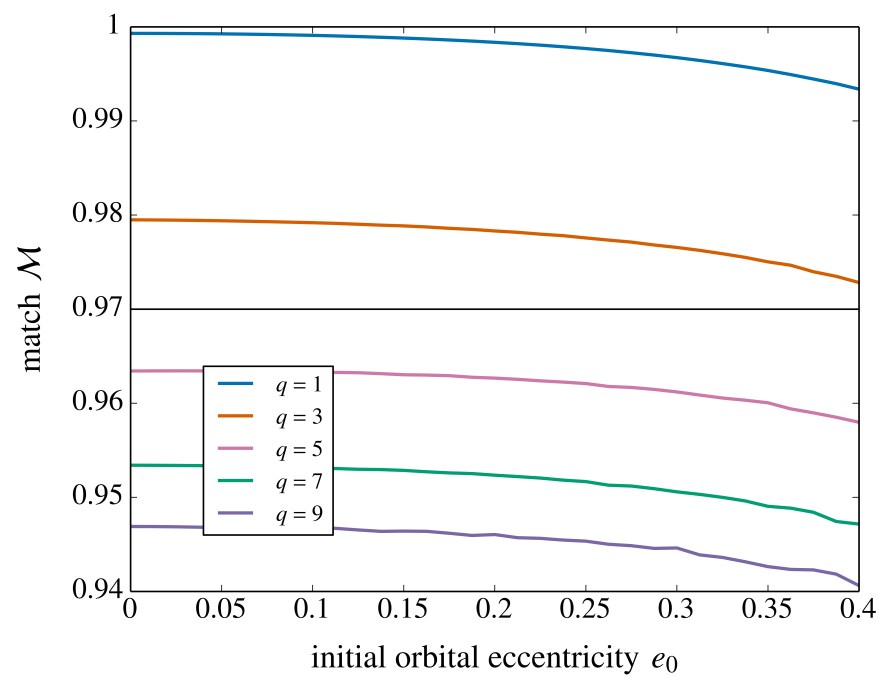

FIG. 3. Matches between eccentric waveform models with Newtonian and 1PN-accurate amplitudes. We consider compact binary systems with a total mass of $m=m_{1}+m_{2}$, with different choices for the mass ratio $q=m_{1} / m_{2}$. As expected, the effect of amplitude corrections on waveform faithfulness is largely independent of the orbital eccentricity $e_{0}$ at $20 \mathrm{~Hz}$. Waveforms with Newtonian amplitudes are faithful representations of amplitude-corrected waveforms only if $q \leq 3$ (blue and orange curves); for higher mass ratios $q \geq 5$ (pink, green and purple curves) the match between waveforms with Newtonian and 1PN-accurate amplitudes falls below the threshold of $\mathcal{M}=0.97$ (denoted by the black line) even in the circular limit.

2. With above inputs, we compute the time domain GW detector response function and express it as a summation of several cosine functions whose arguments are sum of integer multiples of $\phi$ and $\phi^{\prime}$ associated with the orbital and periastron motions. Amplitudes of these functions are expressed in terms of $\omega, e_{t}$ and the angles that specify the antenna patterns $F_{\times}, F_{+}$and the direction of the orbital angular momentum vector. The quadrupolar version of $h(t)$ that explicitly incorporates the next to leading order $e_{t}$ corrections is given by Eq. (2.22) and associated expressions like Eqs. (A1) and (A2). Its $1 P N$ extension is symbolically provided by Eq. 2.32 and the accompanying Mathematica file provide the explicit expressions for various $\mathrm{PN}$ coefficients while incorporating $\mathcal{O}\left(e_{t}^{6}\right)$ corrections.

3. We also provide a prescription to obtain temporally evolving $h(t)$ for compact binaries inspiraling due to $3 \mathrm{PN}$ accurate GW emission along precessing 3PN accurate orbits of moderate eccentricities. This involves imposing temporal evolution for $\omega, e_{t}, \phi^{\prime}$ and $\phi$ with the help of PN accurate differential equations. The relative $3 \mathrm{PN}$ accurate equations for $\omega$ and $e_{t}$ are due to the emission of GWs, as evident from our Eqs. 2.25 and 2.26). The conservative $3 \mathrm{PN}$ accurate differential equation for $\phi^{\prime}$ arises essentially due to periastron advance as evident from Eq. 2.24). The differential equation for $\phi$ is kinematical in nature as $d \phi / d t \equiv \omega$.

4. The structure of the time domain response func- tion allows us to involve the method of stationary phase approximation to compute its Fourier transform. The crucial Fourier phases and the associated 'nine'; stationary points may be concisely written as $\Psi^{ \pm n}(t):=-2 \pi f t+j \phi-(j \pm n) \phi^{\prime}$, where $n$ takes values $0,1,2,3,4$. The nine stationary points, associated with $1 \mathrm{PN}$ accurate amplitude corrected $h(t)$, essentially provide relations between the orbital and Fourier frequencies $F\left(t^{ \pm n}\right)=f /\left(j-(j \pm n) k^{\prime}\right)$, where $k^{\prime}$ is related to the rate of periastron advance per orbit. The explicit expression for the resulting 3PN -accurate Fourier phases with leading order initial eccentricity corrections are provided by Eq. (3.13). Gathering various results, we obtain Eqs. (3.15) and (3.16) that provide the quadrupolar order $h(f)$ while incorporating fourth order orbital eccentricity contributions along with the effects due to $3 \mathrm{PN}$-accurate frequency, eccentricity evolution and periastron advance. Additionally, we have extended these results by including $1 \mathrm{PN}$ accurate amplitude corrections and six order eccentricity contributions.

5. A crucial ingredient to obtain a fully analytic $\tilde{h}(f)$ involves a derivation, detailed in Sec. II, that provides $\mathrm{PN}$ accurate analytic expression for $e_{t}$ in terms of $e_{0}, \omega, \omega_{0}$. We have obtained $3 \mathrm{PN}$ accurate expression for $e_{t}\left(e_{0}, \omega, \omega_{0}\right)$ by extending the post-circular scheme of Refs. [44, 49].

A number of extensions are possible. Influenced by Refs. 72, 73, we are incorporating the effects of leading 
order aligned spin-orbit and spin-spin interactions into these waveforms. It will be interesting to explore data analysis implications of our present waveforms. A possible avenue is to explore the astrophysical implications of using $\mathrm{PN}$-accurate periastron advance contributions that depend both on $m$ and $\eta$, influenced by Refs. [59, 74]. There are on-going efforts to construct analytic IMR templates to model eccentric compact binary coalescence [19, 37]. The present waveform family will be relevant to construct IMR templates for moderately eccentric compact binary mergers which can be used to extract orbital eccentricity and periastron advance as done in Ref [75]. Efforts are on-going to obtain various constructs, using elements of our post-circular Fourier domain approximant, that should allow us to make comparisons with brand new PN-accurate frequency domain waveform family, developed in Refs. 58, 76, for moderate eccentricities.

\section{ACKNOWLEDGEMENTS}

We thank Yannick Boetzel for helpful discussions, suggestions and providing us the lengthy eccentricity enhancement functions. We are grateful to Marc Favata and Blake Moore for their helpful comments. M. H. acknowledges support from Swiss National Science Foundation (SNSF) grant IZCOZ0_177057. We have used software packages from PyCBC [69] and Matplotlib 77] to compute and plot match estimates. 


\section{Appendix A: $\Gamma_{j, \pm n}^{(0)}$ and $\Sigma_{j, \pm n}^{(0)}$ coefficients}

We list the $\Gamma_{j, \pm n}^{(0)}$ and $\Sigma_{j, \pm n}^{(0)}$ coefficients appearing in Eq. 2.21. The relevant $\Gamma_{j, \pm n}^{(0)}$ expressions read

$$
\begin{aligned}
\Gamma_{1,-2}^{(0)} & =F_{+}\left\{\left(\frac{3 e_{t}}{2}-\frac{13 e_{t}^{3}}{16}\right)\left(1+c_{i}^{2}\right) c_{2 \beta}\right\}+F_{\times}\left\{\left(-3 e_{t}+\frac{13 e_{t}^{3}}{8}\right) c_{i} s_{2 \beta}\right\}, \\
\Gamma_{2,-2}^{(0)} & =F_{+}\left\{\left(-2+5 e_{t}^{2}-\frac{23 e_{t}^{4}}{8}\right)\left(1+c_{i}^{2}\right) c_{2 \beta}\right\}+F_{\times}\left\{\left(4-10 e_{t}^{2}+\frac{23 e_{t}^{4}}{4}\right) c_{i} s_{2 \beta}\right\}, \\
\Gamma_{3,-2}^{(0)} & =F_{+}\left\{\left(-\frac{9 e_{t}}{2}+\frac{171 e_{t}^{3}}{16}\right)\left(1+c_{i}^{2}\right) c_{2 \beta}\right\}+F_{\times}\left\{\left(9 e_{t}-\frac{171 e_{t}^{3}}{8}\right) c_{i} s_{2 \beta}\right\}, \\
\Gamma_{4,-2}^{(0)} & =F_{+}\left\{\left(-8 e_{t}^{2}+20 e_{t}^{4}\right)\left(1+c_{i}^{2}\right) c_{2 \beta}\right\}+F_{\times}\left\{\left(16 e_{t}^{2}-40 e_{t}^{4}\right) c_{i} s_{2 \beta}\right\}, \\
\Gamma_{5,-2}^{(0)} & =F_{+}\left\{-\frac{625}{48} e_{t}^{3}\left(1+c_{i}^{2}\right) c_{2 \beta}\right\}+F_{\times}\left\{\frac{625}{24} e_{t}^{3} c_{i} s_{2 \beta}\right\}, \\
\Gamma_{6,-2}^{(0)} & =F_{+}\left\{-\frac{81}{4} e_{t}^{4}\left(1+c_{i}^{2}\right) c_{2 \beta}\right\}+F_{\times}\left\{\frac{81}{2} e_{t}^{4} c_{i} s_{2 \beta}\right\}, \\
\Gamma_{1,0}^{(0)} & =F_{+}\left\{\left(e_{t}-\frac{e_{t}^{3}}{8}\right) s_{i}^{2}\right\}, \\
\Gamma_{2,0}^{(0)} & =F_{+}\left\{\left(e_{t}^{2}-\frac{e_{t}^{4}}{3}\right) s_{i}^{2}\right\}, \\
\Gamma_{2,+2}^{(0)} & =F_{+}\left\{\frac{9}{8} e_{t}^{3} s_{i}^{2}\right\}, \\
\Gamma_{4,0}^{(0)} & =F_{+}\left\{\frac{4}{3} e_{t}^{4} s_{i}^{2}\right\}, \\
\Gamma_{1,+2}^{(0)} & =F_{+}\left\{\frac{7}{48} e_{t}^{3}\left(1+c_{i}^{2}\right) c_{2 \beta}\right\}+F_{\times}\left\{-\frac{7}{24} e_{t}^{3} c_{i} s_{2 \beta}\right\},
\end{aligned}
$$

The $\Sigma_{j, \pm n}^{(0)}$ counterparts of above expressions read

$$
\begin{aligned}
& \Sigma_{1,-2}^{(0)}=F_{+}\left\{\left(\frac{3 e_{t}}{2}-\frac{13 e_{t}^{3}}{16}\right)\left(1+c_{i}^{2}\right) s_{2 \beta}\right\}+F_{\times}\left\{\left(3 e_{t}-\frac{13 e_{t}^{3}}{8}\right) c_{i} c_{2 \beta}\right\}, \\
& \Sigma_{2,-2}^{(0)}=F_{+}\left\{\left(-2+5 e_{t}^{2}-\frac{23 e_{t}^{4}}{8}\right)\left(1+c_{i}^{2}\right) s_{2 \beta}\right\}+F_{\times}\left\{\left(-4+10 e_{t}^{2}-\frac{23 e_{t}^{4}}{4}\right) c_{i} c_{2 \beta}\right\}, \\
& \Sigma_{3,-2}^{(0)}=F_{+}\left\{\left(-\frac{9 e_{t}}{2}+\frac{171 e_{t}^{3}}{16}\right)\left(1+c_{i}^{2}\right) s_{2 \beta}\right\}+F_{\times}\left\{\left(-9 e_{t}+\frac{171 e_{t}^{3}}{8}\right) c_{i} c_{2 \beta}\right\}, \\
& \Sigma_{4,-2}^{(0)}=F_{+}\left\{\left(-8 e_{t}^{2}+20 e_{t}^{4}\right)\left(1+c_{i}^{2}\right) s_{2 \beta}\right\}+F_{\times}\left\{\left(-16 e_{t}^{2}+40 e_{t}^{4}\right) c_{i} c_{2 \beta}\right\}, \\
& \Sigma_{5,-2}^{(0)}=F_{+}\left\{-\frac{625}{48} e_{t}^{3}\left(1+c_{i}^{2}\right) s_{2 \beta}\right\}+F_{\times}\left\{-\frac{625}{24} e_{t}^{3} c_{i} c_{2 \beta}\right\}, \\
& \Sigma_{6,-2}^{(0)}=F_{+}\left\{-\frac{81}{4} e_{t}^{4}\left(1+c_{i}^{2}\right) s_{2 \beta}\right\}+F_{\times}\left\{-\frac{81}{2} e_{t}^{4} c_{i} c_{2 \beta}\right\}, \\
& \Sigma_{1,0}^{(0)}=0 \\
& \Sigma_{2,0}^{(0)}=0 \\
& \Sigma_{3,0}^{(0)}=0
\end{aligned}
$$




$$
\begin{aligned}
\Sigma_{4,0}^{(0)} & =0 \\
\Sigma_{1,+2}^{(0)} & =F_{+}\left\{-\frac{7}{48} e_{t}^{3}\left(1+c_{i}^{2}\right) s_{2 \beta}\right\}+F_{\times}\left\{-\frac{7}{24} e_{t}^{3} c_{i} c_{2 \beta}\right\}, \\
\Sigma_{2,+2}^{(0)} & =F_{+}\left\{-\frac{1}{8} e_{t}^{4}\left(1+c_{i}^{2}\right) s_{2 \beta}\right\}+F_{\times}\left\{-\frac{1}{4} e_{t}^{4} c_{i} c_{2 \beta}\right\} .
\end{aligned}
$$

\section{Appendix B: 3PN accurate $\frac{d \omega}{d t}$ and $\frac{d e_{t}}{d t}$}

We give here the $3 \mathrm{PN}$ accurate expressions for temporal evolution of $\omega$ and $e_{t}$ for obtaining $h(t)$ associated with compact binaries inspiraling along precessing eccentric orbits. $1 \mathrm{PN}$ accurate $\frac{d \omega}{d t}$ and $\frac{d e_{t}}{d t}$ with $\mathcal{O}\left(e_{t}^{6}\right)$ eccentricity corrections are given by Eq. 2.25) and Eq. (2.26) respectively. The $1.5 \mathrm{PN}-3 \mathrm{PN}$ contributions to $\frac{d \omega}{d t}$ appearing in Eq. 2.25 with $\mathcal{O}\left(e_{t}^{6}\right)$ corrections are,

$$
\begin{aligned}
& \dot{\omega}^{1.5 P N}=\pi x^{3 / 2}\left\{4+\frac{2335}{48} e_{t}^{2}+\frac{42955}{192} e_{t}^{4}+\frac{6204647}{9216} e_{t}^{6}\right\} \\
& \dot{\omega}^{2 P N}=x^{2}\left\{\frac{34103}{18144}+\frac{13661}{2016} \eta+\frac{59}{18} \eta^{2}+\left(-\frac{479959}{12096}+\frac{80425}{4032} \eta+\frac{213539}{1728} \eta^{2}\right) e_{t}^{2}+\left(-\frac{2932261}{16128}-\frac{5715083}{16128} \eta\right.\right. \\
& \left.\left.+\frac{2133235}{2304} \eta^{2}\right) e_{t}^{4}+\left(-\frac{19581787}{48384}-\frac{1753627}{768} \eta+\frac{25727065}{6912} \eta^{2}\right) e_{t}^{6}\right\} \\
& \dot{\omega}^{2.5 P N}=\pi x^{5 / 2}\left\{-\frac{4159}{672}-\frac{189}{8} \eta+\left(\frac{7885}{96}-\frac{27645}{56} \eta\right) e_{t}^{2}+\left(\frac{44644883}{43008}-\frac{11707809}{3584} \eta\right) e_{t}^{4}\right. \\
& \left.+\left(\frac{971752501}{193536}-\frac{103819241}{8064} \eta\right) e_{t}^{6}\right\} \\
& \dot{\omega}^{3 P N}=x^{3}\left\{\frac{16447322263}{139708800}+\frac{16 \pi^{2}}{3}-\frac{1712 \gamma}{105}+\left(-\frac{56198689}{217728}+\frac{451 \pi^{2}}{48}\right) \eta+\frac{541}{896} \eta^{2}-\frac{5605}{2592} \eta^{3}-\frac{3424 \log (2)}{105}\right. \\
& -\frac{856 \log (x)}{105}+\left(\frac{277391496167}{139708800}+\frac{992 \pi^{2}}{9}-\frac{106144 \gamma}{315}+\left(-\frac{280153957}{120960}+\frac{188231 \pi^{2}}{2304}\right) \eta-\frac{73109}{448} \eta^{2}\right. \\
& \left.-\frac{6874115}{31104} \eta^{3}-\frac{80464 \log (2)}{315}-\frac{234009 \log (3)}{560}-\frac{53072 \log (x)}{315}\right) e_{t}^{2}+\left(\frac{974308007423}{79833600}+\frac{3059 \pi^{2}}{4}-\frac{46759 \gamma}{20}\right. \\
& +\left(-\frac{33126017}{3780}+\frac{2065129 \pi^{2}}{6144}\right) \eta-\frac{2804209}{32256} \eta^{2}-\frac{114255295}{41472} \eta^{3}-\frac{2730533 \log (2)}{252}+\frac{4446171 \log (3)}{2240} \\
& \left.-\frac{46759 \log (x)}{40}\right) e_{t}^{4}+\left(\frac{150878591021}{3193344}+\frac{76615 \pi^{2}}{24}-\frac{234223 \gamma}{24}+\left(-\frac{7739324653}{362880}+\frac{34978699 \pi^{2}}{36864}\right) \eta\right. \\
& +\frac{21116263}{4608} \eta^{2}-\frac{1935750565}{124416} \eta^{3}+\frac{80906873 \log (2)}{2520}-\frac{134711181 \log (3)}{35840}-\frac{5224609375 \log (5)}{193536} \\
& \left.\left.-\frac{234223 \log (x)}{48}\right) e_{t}^{6}\right\}
\end{aligned}
$$

where $\gamma$ stands for the Euler-Mascheroni constant. The
1.5PN - 3PN contributions to $\frac{d e_{t}}{d t}$ appearing in Eq. 2.26 with $\mathcal{O}\left(e_{t}^{6}\right)$ corrections are, 


$$
\begin{aligned}
& \dot{e}_{t}^{1.5 P N}=\pi x^{3 / 2}\left\{\frac{985}{152}+\frac{21729}{608} e_{t}^{2}+\frac{3061465}{29184} e_{t}^{4}+\frac{161865935}{700416} e_{t}^{6}\right\} \\
& {\dot{e_{t}}}^{2 P N}=x^{2}\left\{-\frac{108197}{38304}+\frac{56407}{4256} \eta+\frac{141}{19} \eta^{2}+\left(-\frac{1368625}{51072}-\frac{288209}{17024} \eta+\frac{274515}{2432} \eta^{2}\right) e_{t}^{2}+\left(-\frac{15037865}{306432}\right.\right. \\
& \left.\left.-\frac{30369109}{102144} \eta+\frac{7578425}{14592} \eta^{2}\right) e_{t}^{4}+\left(-\frac{13488023}{408576}-\frac{65394101}{58368} \eta+\frac{87633595}{58368} \eta^{2}\right) e_{t}^{6}\right\} \text {, } \\
& {\dot{e_{t}}}^{2.5 P N}=\pi x^{5 / 2}\left\{-\frac{55691}{4256}-\frac{19067}{399} \eta+\left(\frac{286789}{3584}-\frac{7810371}{17024} \eta\right) e_{t}^{2}+\left(\frac{535570255}{817152}-\frac{31241795}{16128} \eta\right) e_{t}^{4}\right. \\
& \left.+\left(\frac{92235604259}{39223296}-\frac{164170915723}{29417472} \eta\right) e_{t}^{6}\right\} \\
& \dot{e}_{t}^{3 P N}=x^{3}\left\{\frac{246060953209}{884822400}+\frac{769 \pi^{2}}{57}-\frac{82283 \gamma}{1995}+\left(-\frac{613139897}{2298240}+\frac{22345 \pi^{2}}{3648}\right) \eta-\frac{1046329}{51072} \eta^{2}-\frac{305005}{49248} \eta^{3}\right. \\
& -\frac{11021 \log (2)}{285}-\frac{234009 \log (3)}{5320}-\frac{82283 \log (x)}{3990}+\left(\frac{1316189396351}{589881600}+\frac{14023 \pi^{2}}{114}-\frac{1500461 \gamma}{3990}\right. \\
& +\left(-\frac{5882746699}{4596480}+\frac{46453 \pi^{2}}{1536}\right) \eta-\frac{554719}{4788} \eta^{2}-\frac{100330729}{393984} \eta^{3}-\frac{2271503 \log (2)}{1330}+\frac{6318243 \log (3)}{21280} \\
& \left.-\frac{1500461 \log (x)}{7980}\right) e_{t}^{2}+\left(\frac{1499268531223}{168537600}+\frac{10129 \pi^{2}}{19}-\frac{154829 \gamma}{95}+\left(-\frac{543123237}{170240}+\frac{2360575 \pi^{2}}{29184}\right) \eta\right. \\
& +\frac{36456205}{87552} \eta^{2}-\frac{1523467085}{787968} \eta^{3}+\frac{41683669 \log (2)}{5985}-\frac{281044809 \log (3)}{340480}-\frac{1044921875 \log (5)}{204288} \\
& \left.-\frac{154829 \log (x)}{190}\right) e_{t}^{4}+\left(\frac{682257052877}{26966016}+\frac{976185 \pi^{2}}{608}-\frac{2984337 \gamma}{608}+\left(-\frac{4722976831}{875520}+\frac{24558057 \pi^{2}}{155648}\right) \eta\right. \\
& +\frac{1312493803}{350208} \eta^{2}-\frac{24620050735}{3151872} \eta^{3}-\frac{10971071339 \log (2)}{191520}-\frac{74286859077 \log (3)}{2723840}+\frac{24033203125 \log (5)}{700416} \\
& \left.\left.-\frac{2984337 \log (x)}{1216}\right) e_{t}^{6}\right\} \text {. }
\end{aligned}
$$

\section{Appendix C: 3PN accurate analytic expressions for $e_{t}$ and $\Psi_{j}^{ \pm n}$}

We display explicit expressions for $3 \mathrm{PN}$-accurate $e_{t}$ and Fourier phases that incorporate next to leading order $e_{0}$ corrections at each PN order. These expressions along with Eqs. (3.15), 3.16), 2.31, (A1) and (A2) are required to make operational the fully analytic frequency domain quadrupolar order GW response function for eccentric inspirals that includes $\mathcal{O}\left(e_{0}^{4}\right)$ corrections at every $\mathrm{PN}$ order. We begin by listing explicit expression for the $3 \mathrm{PN}$ accurate $e_{t}$ in terms of $e_{0}, \chi$ and $x$. The underlying computation is detailed in Ref. 49] and requires 3PNaccurate expressions for $\dot{\omega}$ and $\dot{e}_{t}$, given by Eqs. 2.25 and 2.26 . The fully $3 \mathrm{PN}$ accurate $e_{t}$ expression that accounts for all the $\mathcal{O}\left(e_{0}^{3}\right)$ contributions read

$$
e_{t}=\sum_{m=0}^{6} \mathcal{E}_{m} x^{m / 2} .
$$

The coefficients $\mathcal{E}_{m}$ with next to leading order eccentricity corrections $\mathcal{O}\left(e_{0}^{3}\right)$ at each PN order can be listed as,

$$
\mathcal{E}_{0}=e_{0} \chi^{-19 / 18}+\frac{3323}{1824}\left(\chi^{-19 / 18}-\chi^{-19 / 6}\right) e_{0}^{3}
$$


$\mathcal{E}_{1}=0$,

$$
\begin{aligned}
\mathcal{E}_{2}= & \left\{\left(-\frac{2833}{2016}+\frac{197 \eta}{72}\right) \chi^{-19 / 18}+\left(\frac{2833}{2016}-\frac{197 \eta}{72}\right) \chi^{-31 / 18}\right\} e_{0}+\left\{\left(-\frac{9414059}{3677184}+\frac{654631 \eta}{131328}\right) \chi^{-19 / 18}\right. \\
& +\left(\frac{386822573}{47803392}-\frac{1482433 \eta}{131328}\right) \chi^{-31 / 18}+\left(\frac{11412055}{5311488}-\frac{378697 \eta}{43776}\right) \chi^{-19 / 6} \\
& \left.+\left(-\frac{9414059}{1225728}+\frac{654631 \eta}{43776}\right) \chi^{-23 / 6}\right\} e_{0}^{3},
\end{aligned}
$$

$$
\begin{aligned}
\mathcal{E}_{3}= & \left\{\frac{377}{144} \pi\left(-\chi^{-19 / 18}+\chi^{-37 / 18}\right)\right\} e_{0}+\left\{-\frac{1252771 \pi}{262656} \chi^{-19 / 18}+\frac{1315151 \pi}{131328} \chi^{-37 / 18}+\frac{396797 \pi}{43776} \chi^{-19 / 6}\right. \\
& \left.-\frac{1252771 \pi}{87552} \chi^{-25 / 6}\right\} e_{0}^{3}
\end{aligned}
$$

$$
\begin{aligned}
\mathcal{E}_{4}= & \left\{\left(\frac{77006005}{24385536}-\frac{1143767 \eta}{145152}+\frac{43807 \eta^{2}}{10368}\right) \chi^{-19 / 18}+\left(-\frac{8025889}{4064256}+\frac{558101 \eta}{72576}-\frac{38809 \eta^{2}}{5184}\right) \chi^{-31 / 18}\right. \\
& \left.+\left(-\frac{28850671}{24385536}+\frac{27565 \eta}{145152}+\frac{33811 \eta^{2}}{10368}\right) \chi^{-43 / 18}\right\} e_{0}+\left\{\left(\frac{255890954615}{44479217664}-\frac{3800737741 \eta}{264757248}\right.\right. \\
& \left.+\frac{145570661 \eta^{2}}{18911232}\right) \chi^{-19 / 18}+\left(-\frac{1095868349309}{96371638272}+\frac{65400285919 \eta}{1720922112}-\frac{292039301 \eta^{2}}{9455616}\right) \chi^{-31 / 18} \\
& +\left(-\frac{20952382669619}{4047608807424}-\frac{385200824731 \eta}{24092909568}+\frac{4301644427 \eta^{2}}{132378624}\right) \chi^{-43 / 18}+\left(\frac{8180980796033}{1349202935808}\right. \\
& \left.+\frac{14604819923 \eta}{2676989952}-\frac{317361763 \eta^{2}}{14708736}\right) \chi^{-19 / 6}+\left(\frac{32330351815}{3569319936}-\frac{10345778159 \eta}{191213568}+\frac{74603309 \eta^{2}}{1050624}\right) \chi^{-23 / 6} \\
& \left.+\left(-\frac{9164199307}{2118057984}+\frac{1205846917 \eta}{29417472}-\frac{13714021 \eta^{2}}{233472}\right) \chi^{-9 / 2}\right\} e_{0}^{3},
\end{aligned}
$$$$
\mathcal{E}_{5}=\left\{\left(\frac{9901567 \pi}{1451520}-\frac{202589 \pi \eta}{362880}\right) \chi^{-19 / 18}+\left(-\frac{1068041 \pi}{290304}+\frac{74269 \pi \eta}{10368}\right) \chi^{-31 / 18}+\left(-\frac{1068041 \pi}{290304}\right.\right.
$$$$
\left.\left.+\frac{74269 \pi \eta}{10368}\right) \chi^{-37 / 18}+\left(\frac{778843 \pi}{1451520}-\frac{4996241 \pi \eta}{362880}\right) \chi^{-49 / 18}\right\} e_{0}+\left\{\left(\frac{32902907141 \pi}{2647572480}\right.\right.
$$$$
\left.-\frac{673203247 \pi \eta}{661893120}\right) \chi^{-19 / 18}+\left(-\frac{11217854617 \pi}{529514496}+\frac{558877241 \pi \eta}{18911232}\right) \chi^{-31 / 18}+\left(-\frac{3725822783 \pi}{264757248}\right.
$$$$
\left.+\frac{259084747 \pi \eta}{9455616}\right) \chi^{-37 / 18}+\left(\frac{195499289159 \pi}{2647572480}-\frac{65776041763 \pi \eta}{661893120}\right) \chi^{-49 / 18}+\left(-\frac{2057616403 \pi}{32686080}\right.
$$$$
\left.+\frac{2370731599 \pi \eta}{73543680}\right) \chi^{-19 / 6}+\left(\frac{1124125901 \pi}{29417472}-\frac{78169009 \pi \eta}{1050624}\right) \chi^{-23 / 6}+\left(\frac{330949595 \pi}{19611648}\right.
$$$$
\left.\left.-\frac{142768769 \pi \eta}{2101248}\right) \chi^{-25 / 6}+\left(-\frac{12693032573 \pi}{294174720}+\frac{11292740311 \pi \eta}{73543680}\right) \chi^{-29 / 6}\right\} e_{0}^{3}
$$

Due to the lengthy nature of $3 \mathrm{PN}$ order terms in $e_{t}$, we split it in two parts as

$$
\mathcal{E}_{6}=\mathcal{E}_{6}^{\prime} e_{0}+\mathcal{E}_{6}^{\prime \prime} e_{0}^{3},
$$

The explicit form of these two contributions are 


$$
\begin{aligned}
& \mathcal{E}_{6}^{\prime}=\left(-\frac{33320661414619}{386266890240}+\frac{180721 \pi^{2}}{41472}+\frac{3317 \gamma}{252}+\left(\frac{161339510737}{8778792960}+\frac{3977 \pi^{2}}{2304}\right) \eta-\frac{359037739 \eta^{2}}{20901888}+\frac{10647791 \eta^{3}}{2239488}\right. \\
& \left.+\frac{12091 \log (2)}{3780}+\frac{26001 \log (3)}{1120}+\frac{3317 \log (x)}{504}\right) \chi^{-19 / 18}+\left(\frac{218158012165}{49161240576}-\frac{34611934451 \eta}{1755758592}+\frac{191583143 \eta^{2}}{6967296}\right. \\
& \left.-\frac{8629979 \eta^{3}}{746496}\right) \chi^{-31 / 18}-\frac{142129 \pi^{2}}{20736} \chi^{-37 / 18}+\left(\frac{81733950943}{49161240576}-\frac{6152132057 \eta}{1755758592}-\frac{1348031 \eta^{2}}{331776}\right. \\
& \left.+\frac{6660767 \eta^{3}}{746496}\right) \chi^{-43 / 18}+\left(\frac{216750571931393}{2703868231680}+\frac{103537 \pi^{2}}{41472}-\frac{3317 \gamma}{252}+\left(\frac{866955547}{179159040}-\frac{3977 \pi^{2}}{2304}\right) \eta\right. \\
& \left.-\frac{130785737 \eta^{2}}{20901888}-\frac{4740155 \eta^{3}}{2239488}-\frac{12091 \log (2)}{3780}-\frac{26001 \log (3)}{1120}-\frac{3317 \log (x)}{504}-\frac{3317 \log (\chi)}{756}\right) \chi^{-55 / 18} \\
& \mathcal{E}_{6}^{\prime \prime}=\left(-\frac{110724557880778937}{704550807797760}+\frac{600535883 \pi^{2}}{75644928}+\frac{11022391 \gamma}{459648}+\left(\frac{536131194179051}{16012518359040}+\frac{13215571 \pi^{2}}{4202496}\right) \eta\right. \\
& \left.-\frac{1193082406697 \eta^{2}}{38125043712}+\frac{35382609493 \eta^{3}}{4084826112}+\frac{40178393 \log (2)}{6894720}+\frac{28800441 \log (3)}{680960}+\frac{11022391 \log (x)}{919296}\right) \chi^{-19 / 18} \\
& +\left(\frac{29787660990550865}{1165711336538112}-\frac{591234360321013 \eta}{5947506819072}+\frac{107636760191 \eta^{2}}{874119168}-\frac{64940942431 \eta^{3}}{1361608704}\right) \chi^{-31 / 18} \\
& -\frac{495811927 \pi^{2}}{18911232} \chi^{-37 / 18}+\left(\frac{59358100103030627}{8159979355766784}+\frac{2420024232862595 \eta}{291427834134528}-\frac{103398129181999 \eta^{2}}{1156459659264}\right. \\
& \left.+\frac{847423952119 \eta^{3}}{9531260928}\right) \chi^{-43 / 18}+\left(-\frac{3881667007528080426037}{2243994322835865600}+\frac{720177509 \pi^{2}}{75644928}+\frac{517414657 \gamma}{2298240}\right. \\
& +\left(-\frac{1395931720786001359}{1457139170672640}+\frac{295851449 \pi^{2}}{4202496}\right) \eta-\frac{112681906698415 \eta^{2}}{3469378977792}-\frac{1549239851389 \eta^{3}}{28593782784} \\
& \left.+\frac{101727523747 \log (2)}{6894720}-\frac{5477465997 \log (3)}{680960}+\frac{517414657 \log (x)}{4596480}-\frac{517414657 \log (\chi)}{6894720}\right) \chi^{-55 / 18} \\
& +\left(\frac{152896024020300184249}{67999827964723200}-\frac{95207357 \pi^{2}}{8404992}-\frac{245954159 \gamma}{766080}+\left(\frac{12374839994637661}{10793623486464}-\frac{116237911 \pi^{2}}{1400832}\right) \eta\right. \\
& \left.-\frac{3908281091711 \eta^{2}}{128495517696}-\frac{42680326813 \eta^{3}}{1059028992}-\frac{33962745773 \log (2)}{2298240}+\frac{5362264233 \log (3)}{680960}-\frac{245954159 \log (x)}{1532160}\right) \chi^{-19 / 6} \\
& +\left(\frac{23176718595161489}{906664372862976}-\frac{866895029665039 \eta}{32380870459392}-\frac{5814138473063 \eta^{2}}{42831839232}+\frac{62520267311 \eta^{3}}{353009664}\right) \chi^{-23 / 6} \\
& +\frac{149592469 \pi^{2}}{2101248} \chi^{-25 / 6}+\left(-\frac{99813874374700537}{234850269265920}-\frac{429547595 \pi^{2}}{8404992}+\frac{11022391 \gamma}{153216}+\left(-\frac{62659748948903}{1779168706560}\right.\right. \\
& \left.+\frac{13215571 \pi^{2}}{1400832}\right) \eta-\frac{95613034561 \eta^{2}}{1412038656}+\frac{22151672941 \eta^{3}}{151289856}+\frac{40178393 \log (2)}{2298240}+\frac{86401323 \log (3)}{680960}+\frac{11022391 \log (x)}{306432} \\
& \left.-\frac{11022391 \log (\chi)}{459648}\right) \chi^{-31 / 6}+\left(\frac{31472267987495}{6167784849408}-\frac{318662569276073 \eta}{4625838637056}+\frac{4844584781833 \eta^{2}}{18356502528}\right. \\
& \left.-\frac{1562882519 \eta^{3}}{5603328}\right) \chi^{-9 / 2} \text {. }
\end{aligned}
$$

We have pursued careful checking of our results with what is available in Ref. 49] and observed a slight typo in the $\mathcal{O}\left(e_{0}^{5}\right)$ contributions for the $e_{t}$ expression (Eq. (A6e)) of Ref. [49]. The $\eta$ independent term present in the coefficient of $\chi^{-119 / 18}$ should be $16952610560003855 / 162260186038272$ instead of $16633441088056655 / 162260186038272$. Note that the above $e_{t}$ expression is required while computing the Fourier amplitudes $\xi_{j}$. Additionally, it is a crucial ingredient while computing analytic expression for our Fourier phases $\Psi_{j}$. It should be obvious that its frequency dependence is encapsulated in $\chi=F / F_{0}$ and the $\mathrm{PN}$ expansion parameter $x=\left(G m 2 \pi F / c^{3}\right)^{2 / 3}$.

We now display our $3 \mathrm{PN}$ accurate closed form expres- 
sion for the Fourier phases $\Psi_{j}^{ \pm n}$. Recall that nine different Fourier phases appear in our 1PN accurate amplitude corrected $\tilde{h}(f)$ expression, given by Eq. (3.17). To circumvent the task of displaying all the 9 different Fourier phases separately, we provide a general expres- sion for these phases as $\Psi_{j}^{n}$ where $n=0,1,2,3,4$. It is not very difficult to obtain $\Psi_{j}^{ \pm n}$ from $\Psi_{j}^{n}$ by replacing $n$ with appropriate ${ }^{\prime} \pm$ ' sign in the expression. The general expression for $3 \mathrm{PN}$ accurate Fourier phase reads

$$
\Psi_{j}^{n}=\left(j-(j+n) k_{(3)}^{(6)}\right) \phi_{c}-2 \pi f t_{c}-\frac{3 j}{256 \eta x^{5 / 2}} \sum_{m=0}^{6} \mathcal{P}_{m} x^{m / 2}
$$

Various PN coefficients $\mathcal{P}_{m}$ with next to leading order

eccentricity contributions are given by

$$
\begin{aligned}
\mathcal{P}_{0}= & 1-\frac{2355}{1462} e_{0}^{2} \chi^{-19 / 9}+\left(-\frac{2608555}{444448} \chi^{-19 / 9}+\frac{5222765}{998944} \chi^{-38 / 9}\right) e_{0}^{4} \\
\mathcal{P}_{1}= & 0 \\
\mathcal{P}_{2}= & -\frac{2585}{756}-\frac{25 n}{3 j}+\frac{55 \eta}{9}+\left\{\left(\frac{69114725}{14968128}+\frac{1805 n}{172 j}-\frac{128365 \eta}{12432}\right) \chi^{-19 / 9}+\left(-\frac{2223905}{491232}+\frac{154645 \eta}{17544}\right) \chi^{-25 / 9}\right\} e_{0}^{2} \\
& +\left\{\left(\frac{229668231175}{13650932736}+\frac{315685 n}{8256 j}-\frac{426556895 \eta}{11337984}\right) \chi^{-19 / 9}+\left(-\frac{14275935425}{416003328}+\frac{209699405 \eta}{4000032}\right) \chi^{-25 / 9}\right. \\
& +\left(-\frac{259509826776175}{13976341456896}-\frac{225548425 n}{6014496 j}+\frac{1222893635 \eta}{28804608}\right) \chi^{-38 / 9} \\
& \left.+\left(\frac{14796093245}{503467776}-\frac{1028884705 \eta}{17980992}\right) \chi^{-44 / 9}\right\} e_{0}^{4}
\end{aligned}
$$$$
\mathcal{P}_{3}=-16 \pi+\left(\frac{65561 \pi}{4080} \chi^{-19 / 9}-\frac{295945 \pi}{35088} \chi^{-28 / 9}\right) e_{0}^{2}+\left(\frac{217859203 \pi}{3720960} \chi^{-19 / 9}-\frac{3048212305 \pi}{64000512} \chi^{-28 / 9}\right.
$$$$
\left.-\frac{6211173025 \pi}{102085632} \chi^{-38 / 9}+\frac{1968982405 \pi}{35961984} \chi^{-47 / 9}\right) e_{0}^{4}
$$$$
\mathcal{P}_{4}=-\frac{48825515}{508032}-\frac{31805 n}{252 j}+\left(\frac{22105}{504}-\frac{10 n}{j}\right) \eta+\frac{3085 \eta^{2}}{72}+\left\{\left(\frac{115250777195}{2045440512}+\frac{323580365 n}{5040288 j}+\left(-\frac{72324815665}{6562454976}\right.\right.\right.
$$$$
\left.\left.+\frac{36539875 n}{1260072 j}\right) \eta-\frac{10688155 \eta^{2}}{294624}\right) \chi^{-19 / 9}+\left(\frac{195802015925}{15087873024}+\frac{5113565 n}{173376 j}+\left(-\frac{3656612095}{67356576}-\frac{355585 n}{6192 j}\right) \eta\right.
$$$$
\left.\left.+\frac{25287905 \eta^{2}}{447552}\right) \chi^{-25 / 9}+\left(\frac{936702035}{1485485568}+\frac{3062285 \eta}{260064}-\frac{14251675 \eta^{2}}{631584}\right) \chi^{-31 / 9}\right\} e_{0}^{2}+\left\{\left(\frac{382978332618985}{1865441746944}\right.\right.
$$$$
\left.+\frac{1075257552895 n}{4596742656 j}+\left(-\frac{240335362454795}{5984958938112}+\frac{121422004625 n}{1149185664 j}\right) \eta-\frac{35516739065 \eta^{2}}{268697088}\right) \chi^{-19 / 9}
$$$$
+\left(\frac{1256913822951125}{12777273040896}+\frac{1727660975 n}{7727616 j}+\left(-\frac{1182697961961875}{3194318260224}-\frac{25377635 n}{74304 j}\right) \eta+\frac{34290527545 \eta^{2}}{102041856}\right) \chi^{-25 / 9}
$$$$
+\left(-\frac{94372278903235}{7251965779968}+\frac{126823556396665 \eta}{733829870592}-\frac{20940952805 \eta^{2}}{93768192}\right) \chi^{-31 / 9}+\left(-\frac{359074780345285439107}{1705190973672775680}\right.
$$$$
\left.-\frac{100456187745548465 n}{451108723193856 j}+\left(-\frac{41964795442387913}{5074973135930880}-\frac{656130734149165 n}{3717929037312 j}\right) \eta+\frac{203366083643 \eta^{2}}{1130734080}\right) \chi^{-38 / 9}
$$ 


$$
\begin{aligned}
& +\left(-\frac{735191339256903775}{7044076094275584}-\frac{638978688025 n}{3031305984 j}+\left(\frac{55579511401449335}{125787073112064}+\frac{44433039725 n}{108260928 j}\right) \eta\right. \\
& \left.\left.-\frac{240910046095 \eta^{2}}{518482944}\right) \chi^{-44 / 9}+\left(\frac{3654447011975}{98224939008}-\frac{4300262795285 \eta}{18124839936}+\frac{392328884035 \eta^{2}}{1294631424}\right) \chi^{-50 / 9}\right\} e_{0}^{4} \\
& \mathcal{P}_{5}=\frac{14453 \pi}{756}-\frac{32 \pi n}{j}-\frac{65 \pi}{9} \eta-\left(\frac{1675}{756}+\frac{160 n}{3 j}+\frac{65 \eta}{9}\right) \pi \log \left(\frac{f}{j}\right)+\left\{\left(-\frac{458370775 \pi}{6837264}-\frac{4909969 \pi n}{46512 j}\right.\right. \\
& \left.+\frac{15803101 \pi \eta}{229824}\right) \chi^{-19 / 9}+\left(\frac{185734313 \pi}{4112640}-\frac{12915517 \pi \eta}{146880}\right) \chi^{-25 / 9}+\left(\frac{26056251325 \pi}{1077705216}+\frac{680485 \pi n}{12384 j}\right. \\
& \left.\left.-\frac{48393605 \pi \eta}{895104}\right) \chi^{-28 / 9}+\left(-\frac{7063901 \pi}{520128}+\frac{149064749 \pi \eta}{2210544}\right) \chi^{-34 / 9}\right\} e_{0}^{2}+\left\{\left(-\frac{1523166085325 \pi}{6235584768}\right.\right. \\
& \left.-\frac{16315826987 \pi n}{42418944 j}+\frac{52513704623 \pi \eta}{209599488}\right) \chi^{-19 / 9}+\left(\frac{238457223541 \pi}{696563712}-\frac{17513506613 \pi \eta}{33488640}\right) \chi^{-25 / 9} \\
& +\left(\frac{268377522549925 \pi}{1965734313984}+\frac{368891935 \pi n}{1188864 j}-\frac{498450665645 \pi \eta}{1632669696}\right) \chi^{-28 / 9}+\left(-\frac{2408172473789 \pi}{6790791168}\right. \\
& \left.+\frac{992200223893 \pi \eta}{1697697792}\right) \chi^{-34 / 9}+\left(\frac{34901256494241693175 \pi}{79386134731997184}+\frac{84423313781887 \pi n}{193345546752 j}-\frac{15387742160333 \pi \eta}{39404703744}\right) \chi^{-38 / 9} \\
& +\left(-\frac{17596253179825 \pi}{51451158528}+\frac{1223601085925 \pi \eta}{1837541376}\right) \chi^{-44 / 9}+\left(-\frac{7525784976509075 \pi}{38703714803712}-\frac{85031756225 \pi n}{216521856 j}\right. \\
& \left.\left.+\frac{461030900395 \pi \eta}{1036965888}\right) \chi^{-47 / 9}+\left(\frac{14896370333 \pi}{61544448}-\frac{351697861441 \pi \eta}{476969472}\right) \chi^{-53 / 9}\right\} e_{0}^{4}
\end{aligned}
$$

For the ease of presentation, we split the $3 \mathrm{PN}$ contributions to $\Psi_{j}^{n}$ in to three parts

$$
\mathcal{P}_{6}=\mathcal{P}_{6}^{\prime}+\mathcal{P}_{6}^{\prime \prime} e_{0}^{2}+\mathcal{P}_{6}^{\prime \prime \prime} e_{0}^{4}
$$

(C7) Various contributions to $\mathcal{P}_{6}$ are given by,

$$
\begin{aligned}
\mathcal{P}_{6}^{\prime}= & \frac{13966988843531}{4694215680}+\frac{257982425 n}{508032 j}-\frac{640 \pi^{2}}{3}-\frac{6848 \gamma}{21}+\left(-\frac{20562265315}{3048192}-\frac{2393105 n}{1512 j}+\frac{23575 \pi^{2}}{96}\right. \\
& \left.+\frac{1845 \pi^{2} n}{32 j}\right) \eta+\left(\frac{110255}{1728}+\frac{475 n}{24 j}\right) \eta^{2}-\frac{127825 \eta^{3}}{1296}-\frac{13696 \log (2)}{21}-\frac{3424 \log (x)}{21}, \\
\mathcal{P}_{6}^{\prime \prime}= & \left\{\frac{4175723876720788380517}{5556561877278720000}+\frac{534109712725265 n}{2405438042112 j}-\frac{21508213 \pi^{2}}{276480}-\frac{734341 \gamma}{16800}+\left(-\frac{37399145056383727}{28865256505344}\right.\right. \\
& \left.-\frac{1219797059185 n}{2045440512 j}+\frac{12111605 \pi^{2}}{264192}+\frac{639805 n \pi^{2}}{22016 j}\right) \eta+\left(-\frac{159596464273381}{1718170030080}+\frac{43766986495 n}{1022720256 j}\right) \eta^{2}-\frac{69237581 \eta^{3}}{746496} \\
& \left.-\frac{9663919 \log (2)}{50400}+\frac{4602177 \log (3)}{44800}-\frac{734341 \log (x)}{33600}\right\} \chi^{-19 / 9}+\left\{\frac{326505451793435}{2061804036096}+\frac{916703174045 n}{5080610304 j}\right. \\
& \left.-\left(\frac{13467050491570355}{39689727694848}+\frac{9519440485 n}{35282016 j}\right) \eta-\left(\frac{2186530635995}{52499639808}+\frac{7198355375 n}{45362592 j}\right) \eta^{2}+\frac{2105566535 \eta^{3}}{10606464}\right\} \chi^{-25 / 9} \\
& +\frac{24716497 \pi^{2}}{293760} \chi^{-28 / 9}+\left\{-\frac{82471214720975}{45625728024576}-\frac{2153818055 n}{524289024 j}+\left(-\frac{48415393035455}{1629490286592}-\frac{119702185 n}{1560384 j}\right) \eta\right. \\
& \left.+\left(\frac{906325428545}{6466231296}+\frac{32769775 n}{222912 j}\right) \eta^{2}-\frac{2330466575 \eta^{3}}{16111872}\right\} \chi^{-31 / 9}+\left\{-\frac{4165508390854487}{16471063977984}-\frac{96423905 \pi^{2}}{5052672}\right.
\end{aligned}
$$




$$
\begin{aligned}
& +\frac{2603845 \gamma}{61404}+\left(-\frac{1437364085977}{53477480448}+\frac{3121945 \pi^{2}}{561408}\right) \eta+\frac{4499991305 \eta^{2}}{636636672}+\frac{2425890995 \eta^{3}}{68211072}+\frac{1898287 \log (2)}{184212} \\
& \left.+\frac{12246471 \log (3)}{163744}+\frac{2603845 \log (x)}{122808}-\frac{2603845 \log (\chi)}{184212}\right\} \chi^{-37 / 9}, \\
& \mathcal{P}_{6}^{\prime \prime \prime}=\left\{\frac{13875930442343179788457991}{5067584432078192640000}+\frac{1774846575386055595 n}{2193759494406144 j}-\frac{71471791799 \pi^{2}}{252149760}-\frac{2440215143 \gamma}{15321600}\right. \\
& +\left(-\frac{124277359022363124821}{26325113932873728}-\frac{4053385627671755 n}{1865441746944 j}+\frac{40246863415 \pi^{2}}{240943104}+\frac{2126072015 n \pi^{2}}{20078592 j}\right) \eta \\
& +\left(-\frac{530339050780445063}{1566971067432960}+\frac{7654615585415 n}{49090572288 j}\right) \eta^{2}-\frac{230076481663 \eta^{3}}{680804352}-\frac{32113202837 \log (2)}{45964800} \\
& \left.+\frac{5097678057 \log (3)}{13619200}-\frac{2440215143 \log (x)}{30643200}\right\} \chi^{-19 / 9}+\left\{\frac{2095939685244436475}{1746053475139584}+\frac{5884601777755325 n}{4302551126016 j}\right. \\
& +\left(-\frac{17381974915387486205}{8402882349109248}-\frac{527634379756765 n}{358545927168 j}\right) \eta+\left(-\frac{386694251193132845}{933653594345472}-\frac{9761006428375 n}{10342670976 j}\right) \eta^{2} \\
& \left.+\frac{2855158909615 \eta^{3}}{2418273792}\right\} \chi^{-25 / 9}+\frac{254578148953 \pi^{2}}{535818240} \chi^{-28 / 9}+\left\{\frac{141251897794072110575}{3786570420215611392}+\frac{194154433667165 n}{2290094456832 j}\right. \\
& +\left(-\frac{11182467092862313645}{19319236837834752}-\frac{15348073704055 n}{13631514624 j}\right) \eta+\left(\frac{1038816664853665}{594291769344}+\frac{2534255435 n}{1741824 j}\right) \eta^{2} \\
& \left.-\frac{147245442666235 \eta^{3}}{102858190848}\right\} \chi^{-31 / 9}+\left\{\frac{102453749612934666311}{19868699733442560}-\frac{598067688595 \pi^{2}}{4608036864}-\frac{36290762107 \gamma}{56000448}\right. \\
& +\left(\frac{6738669506224179365}{2219101528670208}-\frac{110934582115 \pi^{2}}{512004096}\right) \eta-\frac{1484623162301215 \eta^{2}}{6604468835328}+\frac{128895671353745 \eta^{3}}{217729741824} \\
& \left.-\frac{1140350944327 \log (2)}{24000192}+\frac{1296725746149 \log (3)}{49778176}-\frac{36290762107 \log (x)}{112000896}+\frac{36290762107 \log (\chi)}{168001344}\right\} \chi^{-37 / 9} \\
& +\left\{-\frac{3123488330286080905561719773}{355085641155718958284800}-\frac{85280660877506238107 n}{124770071244349440 j}+\frac{300051120571 \pi^{2}}{970776576}+\frac{211649317 \gamma}{191520}\right. \\
& +\left(-\frac{40336854286157147692937}{32939298808508252160}+\frac{584462420500316711 n}{495119330334720 j}+\frac{2786391039419 \pi^{2}}{17972849664}-\frac{91683875075 n \pi^{2}}{1089263616 j}\right) \eta \\
& +\left(\frac{14654969487690651143}{35648591784099840}-\frac{46042929781519 n}{107385626880 j}\right) \eta^{2}+\frac{49171400252465 \eta^{3}}{91738386432}+\frac{2117998887803 \log (2)}{44241120} \\
& \left.-\frac{334711679031 \log (3)}{13108480}+\frac{211649317 \log (x)}{383040}\right\} \chi^{-38 / 9}+\left\{-\frac{1017258852718193648990131}{859416250731078942720}\right. \\
& -\frac{284592379883138801345 n}{227358796489703424 j}+\left(\frac{69311096542161812013731}{30693437526109962240}+\frac{17602484074819772515 n}{12179935526234112 j}\right) \eta \\
& \left.+\left(\frac{3272123415010135297}{2970715982008320}+\frac{129257754627385505 n}{66922722671616 j}\right) \eta^{2}-\frac{40063118477671 \eta^{3}}{20353213440}\right\} \chi^{-44 / 9} \\
& -\frac{2341612230425 \pi^{2}}{3675082752} \chi^{-47 / 9}+\left\{-\frac{181582918442691290125}{1374276523167055872}-\frac{157819616198875 n}{591398019072 j}+\left(\frac{1741702918744309017425}{1521520436363526144}\right.\right. \\
& \left.\left.+\frac{185709581143825 n}{109127015424 j}\right) \eta+\left(-\frac{18130335399490218365}{6037779509379072}-\frac{16942972137575 n}{7794786816 j}\right) \eta^{2}+\frac{91862546967565 \eta^{3}}{37330771968}\right\} \chi^{-50 / 9} \\
& +\left\{\frac{259620437372696563}{159257838845952}+\frac{691917129965 \pi^{2}}{2589262848}-\frac{558835855 \gamma}{2030112}+\left(-\frac{245999063921173}{13702378991616}-\frac{20770936405 \pi^{2}}{575391744}\right) \eta\right. \\
& +\frac{255806950720535 \eta^{2}}{326247118848}-\frac{9022269087085 \eta^{3}}{8738762112}-\frac{12629690323 \log (2)}{188800416}-\frac{27159422553 \log (3)}{55940864}-\frac{558835855 \log (x)}{4060224} \\
& \left.+\frac{558835855 \log (\chi)}{6090336}\right\} \chi^{-56 / 9}
\end{aligned}
$$


Let us emphasize that the above expression indeed provides all the required Fourier phases, $\Psi_{j}^{ \pm n}$ 's that appear in Eq. 3.17 for $\tilde{h}(f)$. For instance, Fourier phases present in the quadrupolar order $\tilde{h}(f)$, namely, $\Psi_{j}^{0}, \Psi_{j}^{+2}$ and $\Psi_{j}^{-2}$ are obtained by putting in Eq. C5 $n=0,+2,-2$, respectively. Further, one should evaluate these Fourier phases at the correct stationary points and this requires us to use $x=\left\{\frac{G m 2 \pi f}{c^{3}\left(j-(j \pm n) k_{(3)}^{(6)}\right)}\right\}^{2 / 3}$. We note in passing that the $3 \mathrm{PN}$ accurate $e_{t}$ and $\Psi_{j}^{n}$ expressions along with $1 \mathrm{PN}$ accurate Fourier amplitudes while incorporating eccentricity corrections to $\mathcal{O}\left(e_{0}^{6}\right)$ at each PN order can be found in the attached Mathematica notebook.
[1] B. P. Abbott, R. Abbott, T. D. Abbott, M. R. Abernathy, F. Acernese, K. Ackley, C. Adams, T. Adams, P. Addesso, R. X. Adhikari, et al., Physical Review Letters 116, 131103 (2016), 1602.03838.

[2] F. Acernese, M. Agathos, K. Agatsuma, D. Aisa, N. Allemandou, A. Allocca, J. Amarni, P. Astone, G. Balestri, G. Ballardin, et al., Classical and Quantum Gravity 32, 024001 (2015), 1408.3978.

[3] B. P. Abbott, R. Abbott, T. D. Abbott, M. R. Abernathy, F. Acernese, K. Ackley, C. Adams, T. Adams, P. Addesso, R. X. Adhikari, et al., Physical Review Letters 116, 061102 (2016), 1602.03837.

[4] B. P. Abbott, R. Abbott, T. D. Abbott, M. R. Abernathy, F. Acernese, K. Ackley, C. Adams, T. Adams, P. Addesso, R. X. Adhikari, et al., Physical Review Letters 116, 241103 (2016), 1606.04855.

[5] B. P. Abbott, R. Abbott, T. D. Abbott, F. Acernese, K. Ackley, C. Adams, T. Adams, P. Addesso, R. X. Adhikari, V. B. Adya, et al., Physical Review Letters 118, 221101 (2017), 1706.01812.

[6] B. P. Abbott, R. Abbott, T. D. Abbott, F. Acernese, K. Ackley, C. Adams, T. Adams, P. Addesso, R. X. Adhikari, V. B. Adya, et al., ApJ Letters 851, L35 (2017), 1711.05578.

[7] B. P. Abbott, R. Abbott, T. D. Abbott, F. Acernese, K. Ackley, C. Adams, T. Adams, P. Addesso, R. X. Adhikari, V. B. Adya, et al., Physical Review Letters 119, 141101 (2017), 1709.09660.

[8] B. P. Abbott, R. Abbott, T. D. Abbott, F. Acernese, K. Ackley, C. Adams, T. Adams, P. Addesso, R. X. Adhikari, V. B. Adya, et al., Physical Review Letters 119, 161101 (2017), 1710.05832.

[9] The LIGO Scientific Collaboration and the Virgo Collaboration, arXiv e-prints arXiv:1811.12907 (2018).

[10] K. Belczynski, D. E. Holz, T. Bulik, and R. O'Shaughnessy, Nature (London) 534, 512 (2016), 1602.04531 .

[11] D. Park, C. Kim, H. M. Lee, Y.-B. Bae, and K. Belczynski, MNRAS 469, 4665 (2017), 1703.01568.

[12] B.-M. Hoang, S. Naoz, B. Kocsis, F. A. Rasio, and F. Dosopoulou, Astrophys. J. 856, 140 (2018), 1706.09896 .

[13] B. McKernan, K. E. S. Ford, J. Bellovary, N. W. C. Leigh, Z. Haiman, B. Kocsis, W. Lyra, M.-M. Mac Low, B. Metzger, M. O'Dowd, et al., Astrophys. J. 866, 66 (2018), 1702.07818.

[14] K. Kremer, S. Chatterjee, K. Breivik, C. L. Rodriguez, S. L. Larson, and F. A. Rasio, Phys. Rev. Lett. 120, 191103 (2018), 1802.05661.

[15] C. L. Rodriguez, M. Zevin, C. Pankow, V. Kalogera, and F. A. Rasio, ApJ Letters 832, L2 (2016), 1609.05916.
[16] X. Chen and P. Amaro-Seoane, ApJ Letters 842, L2 (2017), 1702.08479.

[17] A. Nishizawa, A. Sesana, E. Berti, and A. Klein, MNRAS 465, 4375 (2017), 1606.09295.

[18] B. P. Abbott, R. Abbott, T. D. Abbott, M. R. Abernathy, F. Acernese, K. Ackley, C. Adams, T. Adams, P. Addesso, R. X. Adhikari, et al., Physical Review Letters 116, 241102 (2016), 1602.03840.

[19] E. A. Huerta, P. Kumar, B. Agarwal, D. George, H.Y. Schive, H. P. Pfeiffer, R. Haas, W. Ren, T. Chu, M. Boyle, et al., Phys. Rev. D 95, 024038 (2017), 1609.05933.

[20] C. L. Rodriguez, S. Chatterjee, and F. A. Rasio, Phys. Rev. D 93, 084029 (2016), 1602.02444.

[21] J. Samsing, M. MacLeod, and E. Ramirez-Ruiz, Astrophys. J. 784, 71 (2014), 1308.2964.

[22] J. Samsing and E. Ramirez-Ruiz, Astrophys. J. 840, L14 (2017), 1703.09703.

[23] C. L. Rodriguez, P. Amaro-Seoane, S. Chatterjee, and F. A. Rasio, Physical Review Letters 120, 151101 (2018), 1712.04937.

[24] J. Samsing, Phys. Rev. D 97, 103014 (2018), 1711.07452.

[25] C. L. Rodriguez, P. Amaro-Seoane, S. Chatterjee, K. Kremer, F. A. Rasio, J. Samsing, C. S. Ye, and M. Zevin, Phys. Rev. D 98, 123005 (2018), 1811.04926.

[26] R. M. OLeary, Y. Meiron, and B. Kocsis, The Astrophysical Journal Letters 824, L12 (2016), URL http: //stacks.iop.org/2041-8205/824/i=1/a=L12

[27] L. Randall and Z.-Z. Xianyu, Astrophys. J. 864, 134 (2018), 1802.05718.

[28] M. Zevin, J. Samsing, C. Rodriguez, C.-J. Haster, and E. Ramirez-Ruiz, ArXiv e-prints (2018), 1810.00901.

[29] L. Gondán, B. Kocsis, P. Raffai, and Z. Frei, Astrophys. J. 855, 34 (2018), 1705.10781.

[30] L. Gondán and B. Kocsis, arXiv e-prints arXiv:1809.00672 (2018), 1809.00672.

[31] S. Burke-Spolaor, S. R. Taylor, M. Charisi, T. Dolch, J. S. Hazboun, A. M. Holgado, L. Z. Kelley, T. J. W. Lazio, D. R. Madison, N. McMann, et al., arXiv e-prints arXiv:1811.08826 (2018), 1811.08826.

[32] M. Bonetti, A. Sesana, F. Haardt, E. Barausse, and M. Colpi, arXiv e-prints arXiv:1812.01011 (2018), 1812.01011 .

[33] I. Hinder, F. Herrmann, P. Laguna, and D. Shoemaker, Phys. Rev. D 82, 024033 (2010), 0806.1037.

[34] T. Damour, A. Gopakumar, and B. R. Iyer, Phys. Rev. D 70, 064028 (2004), gr-qc/0404128.

[35] C. Königsdörffer and A. Gopakumar, Phys. Rev. D 73, 124012 (2006), URL https://link.aps.org/doi/ 10.1103/PhysRevD.73.124012

[36] I. Hinder, L. E. Kidder, and H. P. Pfeiffer, Phys. Rev. D 
98, 044015 (2018), 1709.02007.

[37] E. A. Huerta, C. J. Moore, P. Kumar, D. George, A. J. K. Chua, R. Haas, E. Wessel, D. Johnson, D. Glennon, A. Rebei, et al., Phys. Rev. D 97, 024031 (2018), 1711.06276.

[38] T. Damour and A. Nagar, in Lecture Notes in Physics, Berlin Springer Verlag, edited by F. Haardt, V. Gorini, U. Moschella, A. Treves, and M. Colpi (2016), vol. 905 of Lecture Notes in Physics, Berlin Springer Verlag, p. 273.

[39] T. Hinderer and S. Babak, Phys. Rev. D 96, 104048 (2017), 1707.08426.

[40] Z. Cao and W.-B. Han, Phys. Rev. D 96, 044028 (2017), 1708.00166.

[41] W. E. East, S. T. McWilliams, J. Levin, and F. Pretorius, Phys. Rev. D 87, 043004 (2013), 1212.0837.

[42] V. Tiwari, S. Klimenko, N. Christensen, E. A. Huerta, S. R. P. Mohapatra, A. Gopakumar, M. Haney, P. Ajith, S. T. McWilliams, G. Vedovato, et al., Phys. Rev. D 93, 043007 (2016), 1511.09240.

[43] A. Buonanno, B. R. Iyer, E. Ochsner, Y. Pan, and B. S. Sathyaprakash, Phys. Rev. D 80, 084043 (2009), 0907.0700.

[44] N. Yunes, K. G. Arun, E. Berti, and C. M. Will, Phys. Rev. D 80, 084001 (2009), 0906.0313.

[45] Y. Boetzel, A. Susobhanan, A. Gopakumar, A. Klein, and P. Jetzer, Phys. Rev. D 96, 044011 (2017), 1707.02088 .

[46] M. Tessmer and A. Gopakumar, MNRAS 374, 721 (2007), gr-qc/0610139.

[47] R.-M. Memmesheimer, A. Gopakumar, and G. Schäfer, Phys. Rev. D 70, 104011 (2004), gr-qc/0407049.

[48] A. Królak, K. D. Kokkotas, and G. Schäfer, Phys. Rev. D 52, 2089 (1995), gr-qc/9503013.

[49] S. Tanay, M. Haney, and A. Gopakumar, Phys. Rev. D 93, 064031 (2016), 1602.03081.

[50] K. G. Arun, L. Blanchet, B. R. Iyer, and S. Sinha, Phys. Rev. D 80, 124018 (2009), 0908.3854.

[51] A. Klein, Y. Boetzel, A. Gopakumar, P. Jetzer, and L. de Vittori, Phys. Rev. D 98, 104043 (2018), 1801.08542.

[52] T. Damour, B. R. Iyer, and B. S. Sathyaprakash, Phys. Rev. D 57, 885 (1998), URL https://link.aps.org/ doi/10.1103/PhysRevD.57.885

[53] K. S. Thorne, Three Hundred Years of Gravitation (1987), pp. 330-458.

[54] P. Colwell, Solving Kepler's equation over three centuries (1993).

[55] P. C. Peters and J. Mathews, Phys. Rev. 131, 435 (1963), URL https://link.aps.org/doi/10.1103/ PhysRev.131.435.

[56] P. C. Peters, Phys. Rev. 136, B1224 (1964), URL https : //link.aps.org/doi/10.1103/PhysRev.136.B1224.

[57] W. Junker and G. Schaefer, MNRAS 254, 146 (1992).
[58] B. Moore, T. Robson, N. Loutrel, and N. Yunes, Class. Quant. Grav. 35, 235006 (2018), 1807.07163.

[59] B. Mikóczi, B. Kocsis, P. Forgács, and M. Vasúth, Phys. Rev. D 86, 104027 (2012), 1206.5786.

[60] V. Pierro, I. M. Pinto, and A. D. A. M. Spallicci di F., Monthly Notices of the Royal Astronomical Society 334, 855 (2002).

[61] V. Pierro, I. Pinto, A. Spallicci, E. Laserra, and F. Recano, Monthly Notices of the Royal Astronomical Society 325, $358 \quad$ (2001), ISSN 0035-8711, http://oup.prod.sis.lan/mnras/articlepdf/325/1/358/2833488/325-1-358.pdf, URL https : //doi.org/10.1046/j.1365-8711.2001.04442.x

[62] C. M. Bender and S. A. Orszag, Advanced mathematical methods for scientists and engineers (Springer, New York, 1999).

[63] B. Moore, M. Favata, K. G. Arun, and C. K. Mishra, Phys. Rev. D93, 124061 (2016), 1605.00304.

[64] L. Blanchet, T. Damour, and B. R. Iyer, Phys. Rev. D 51, 5360 (1995), gr-qc/9501029.

[65] L. Blanchet, B. R. Iyer, C. M. Will, and A. G. Wiseman, Classical and Quantum Gravity 13, 575 (1996), gr-qc/9602024.

[66] C. Moreno-Garrido, J. Buitrago, and E. Mediavilla, MNRAS 266, 16 (1994).

[67] E. Mediavilla, J. Buitrago, and C. Moreno-Garrido, Monthly Notices of the Royal Astronomical Society 274, 115 (1995).

[68] G. M. Harry and LIGO Scientific Collaboration, Classical and Quantum Gravity 27, 084006 (2010).

[69] A. Nitz, I. Harry, D. Brown, C. M. Biwer, J. Willis, T. D. Canton, L. Pekowsky, C. Capano, T. Dent, A. R. Williamson, et al., gwastro/pycbc: Pre-o3 release $v 1$ (2019), URL https://doi.org/10.5281/zenodo. 2556644

[70] J. Creighton and W. Anderson, Gravitational-Wave Physics and Astronomy: An Introduction to Theory, Experiment and Data Analysis. (2011).

[71] C. Van Den Broeck and A. S. Sengupta, Classical and Quantum Gravity 24, 155 (2007), gr-qc/0607092.

[72] C. Königsdörffer and A. Gopakumar, Phys. Rev. D 71, 024039 (2005), gr-qc/0501011.

[73] L. E. Kidder, Phys. Rev. D 52, 821 (1995), URL https: //link.aps.org/doi/10.1103/PhysRevD.52.821.

[74] N. Seto, Phys. Rev. Lett. 87, 251101 (2001), URL https://link.aps.org/doi/10.1103/PhysRevLett.87. 251101

[75] A. H. Mroué, H. P. Pfeiffer, L. E. Kidder, and S. A. Teukolsky, Phys. Rev. D 82, 124016 (2010), URL https: //link.aps.org/doi/10.1103/PhysRevD.82.124016

[76] B. Moore and N. Yunes, arXiv:1903.05203 (2019).

[77] J. D. Hunter, Computing In Science \& Engineering 9, 90 (2007). 